\title{
The Hidden Symmetries of Multi-Centre Metrics
}

\author{
G. W. Gibbons * and P. J. Ruback \\ Laboratoire de Physique Théorique de l'Ecole Normale Supérieure ${ }^{\star \star}, 24$ rue Lhomond, \\ F-75231 Paris Cedex 05, France \\ Groupc d'Astrophysique Relativiste - Observatoire de Paris-Meudon
}

\begin{abstract}
We discuss the dynamical symmetries of the multi-centre metrics and apply our results to the scattering of B.P.S. monopoles and fluctuations around such monopoles. In particular, we give a detailed account of the hidden symmetries of the Taub-NUT metric.
\end{abstract}

\section{Introduction}

Understanding the dynamics of solutions of non-linear field equations is an important, but difficult, problem for modern physical theories. For some very special theories in $1+1$ dimensions (e.g. Sine-Gordon equation) this problem can be treated exactly, but for other systems a complete discussion is not as yet available. A typical first step in such theories is to consider static stable solutions as "solitons". Once these have been obtained, however, one is still far from discussing the interactions of the objects that these solutions represent. About ten years ago these solutions were incorporated into quantum field theory [1] and their rôle in these theories in a perturbative expansion in powers of the inverse soliton mass was discussed extensively; however, at that time no attempt was made to consider the interactions of solitons with each other - an intrinsically non-linear problem.

Later Manton [2] observed that for a certain class of multi-monopole solutions (the B.P.S. monopoles of Yang-Mills-Higgs theories) some progress was possible as the low energy dynamics could be reasonably described by only a finite number of naturally obtained parameters. He argued that these solutions would evolve according to a geodesic motion on the parameter space of static multimonopole solutions, with metric obtained from the restriction of the kinetic energy functional to this submanifold of the configuration space of fields. This identifi-

\footnotetext{
* Permanent address: Department of Applied Mathematics and Theoretical Physics, Cambridge University, United Kingdom

$\star \star$ Laboratoire Propre du Centre National de la Recherche Scientifique, associć à l'Ecole Normale Supérieure et à l'Université de Paris-Sud, France
} 
cation of the important degrees of freedom meant that further advances were possible.

Subsequent work [3] revealed that this metric was hyperKähler and in the case of 2 monopoles could be found uniquely as a result of the natural geometric symmetries of the physical problem. The metric is called the Atiyah-Hitchin metric. It has one free parameter which may be identified with physical constants by considering the asymptotic form of the metric and comparing it with long range monopole interaction forces [4].

The dynamics of both the full Atiyah-Hitchin geodesic motion and the asymptotic form of the problem have been thoroughly described in [5]. In particular, the work in [5] brought to light an interesting and useful generalization of the conserved Runge-Lenz vectors in the standard Coulomb problem.

In this paper we wish to amplify and extend the results about the Runge-Lenz vector which were announced in our recent letter [6]. We also wish to give some general results which were useful in discussing the issues raised in the monopole motion problem and which may prove useful in other systems, possibly generalized, for example in the case of higher dimensional hyperKähler metrics.

The asymptotic form of the Atiyah-Hitchin metric is the Taub-NUT metric with a negative mass parameter. When discussing the geodesic equations in this asymptotic metric the existence of extra conserved quantities was noticed. These reflect a symmetry of the system's phase space (i.e. of the relevant Hamiltonian) and enable the Schrödinger equation to be separated in a special coordinate system. This is related to the existence of a Killing tensor of rank two on Taub-NUT. A major motivation for discussing this problem in detail is to discover to what extent these extra symmetries and separability persist for the full Atiyah-Hitchin case and for other "generalized multi-centre metrics" which are relevant to other problems in monopole physics. The many connections between the theory of separability, the existence of special tensors - Killing-Yano and Killing tensors - and the Petrov type of metrics require clarification.

The main features of the Taub-NUT metric relevant here are the fact that it possesses a self-dual Riemann tensor, a self-dual Killing vector and a Killing-Yano tensor. It also sits at a special point as the intersection of 3 families of Ricci flat metrics. We give a description of these families and establish some new results and give simplified proofs of some known results relevant to them. These results have been useful to us, not only in relation to this work, but in relation to the identification of other 4 dimensional hyperKähler metrics. This is of some importance in the Harmonic Superspace construction of hyperKähler metrics [38].

We also give necessary conditions for the existence of Killing-Yano two forms. Combining these results with previous work leads us to believe that the AtiyahHitchin metric does not possess the simplifications for the geodesic problem that we obtain in the Taub-NUT limit.

The rest of this paper is organized as follows:

In Sect. 2 we describe some relevant properties of 3 useful classes of Ricci-flat 4 metrics - the multi-centre metrics, the Taub-NUT metrics, and the $S O(3)$ invariant self-dual metrics. We have tried to give a formal systematic picture of the relation between these classes and of metrics and their properties. In particular, the most important information for our purposes is whether the action of the isometry 
group or its subgroups is holomorphic with respect to any of the preferred complex structures. This information provides a powerful characterization of the metrics which allows us in the later part of this paper to argue that the Atiyah-Hitchin metric does not possess similar conserved quantities to those in Taub-NUT. It is also important in other contexts, for example when discussing the construction of hyperKähler metrics using supersymmetric methods. For this reason we have tried to spell out rather explicitly some results which are perhaps contained rather implicitly elsewhere in the literature. For the same reason we have organized the material in this section in a rather formal way to enable the reader to obtain the relevant facts quickly giving self contained proofs of both new and old results.

Section 3 contains a brief summary of the properties of the conserved quantities discovered in [5] and relates these to the existence on the self-dual Taub-NUT metric of 2 important geometric structures: three symmetric second rank Killing tensors, $K_{\alpha \beta}^{i}$ and a skew symmetric second rank Killing-Yano tensor $Y_{\alpha \beta}$. These two structures are linked by the existence of the 3 complex structures associated with the hyperKähler metric.

In Sect. 4 we describe what is known of the separability of the Schrödinger equation in the multi-centre metrics. In particular, we obtain a new result: the separability of the Schrödinger equation in the 2-centre metric. This separability may be viewed as giving rise to the existence of symmetric Killing tensors. We also discuss a generalization of the Schrödinger equation which is relevant for the fluctuations around the B.P.S. monopoles and find it to be separable as well. Various previously observed separation and symmetry properties of the multicentre metrics are shown to arise as special cases.

In Sect. 5 we treat Killing-Yano tensors. In particular, we show that their existence requires the Weyl tensor to be of Petrov type $D$. The existence of a Yano tensor gives rise to associated Killing vectors. These are identified in our case and we are able to show that this situation is very special to the Taub-NUT case. As with Sect. 2 and to a lesser extent Sect. 4 we have laid out in a systematic and formal way various results (some of them known already) about Yano tensors together with self-contained proofs.

One aspect of the existence of hidden symmetries is that we can reduce our problem to that of a simple harmonic oscillator provided we use an appropriate set of coordinates. In preparation for the treatment of our generalized Coulomb problem we give in Sect. 6 a brief review of the properties of the standard isotropic oscillator. In particular, we describe the various important group actions and their holomorphic properties.

In Sect. 7 we return to our specific problem and identify the action of the conformal group (or its covering) $S U(2,2)$. We outline how this is related to the twistor formalism. We find some results of Mack and Todorov to be especially helpful in this respect. We then show that, for the bound states, there exists a family of $S U(2) \times S U(2)$ subgroups of $S U(2,2)$ which act transitively on the set of classical orbits having fixed energy and electric charge. This action is described in some detail. The diagonal $S U(2)$ subgroup corresponds to the manifest geometric symmetries, the remaining elements constitute the "hidden symmetries."

The existence of hidden symmetries is also known for the fluctuations around the asymptotic Wu-Yang limit of the spherically symmetric B.P.S. monopole. In 
Sect. 8 we are able to generalize this result by showing that the large distance fluctuations around a multi-monopole are governed by a Schrödinger type equation on the multi-centre metrics.

Finally, in Sect. 9 we point out that because the Atiyah-Hitchin metric has nontrivial second homology it is possible to introduce a connection on the 2 monopole moduli-space which enters the generalized Schrödinger equation. We describe some of the modifications of previous results this brings about. Finally, in Sect. 10 we point out the irrelevance of Killing tensors for $\sigma$-models with targets admitting them.

\section{The Half-Flat Taub-NUT Metrics}

It is helpful to consider the half-flat Taub-NUT metrics as special cases of 3 classes of Ricci flat 4-metrics.

1) The multi-centre metrics;

2) The general, $U(2)$ invariant, Taub-NUT family;

3) The $S O(3)$ invariant half-flat metrics.

In this way we may regard some of its special properties as being inherited from a more general class of metrics. Other of its special properties, for example its extra "hidden" symmetries, may be regarded as arising from the way one approaches it as a special case of the more general metrics.

(2.1). The Multi-Centre Metrics. These are conveniently characterized by the following (local) theorem:

Theorem 2.1. Let $\left(M, g_{\alpha \beta}\right)$ be a Ricci flat Riemannian 4-metric with an $\varepsilon$-self-dual Killing vector $q^{\alpha} \frac{\partial}{\partial x^{\alpha}}=\frac{\partial}{\partial \tau}$,

$$
q_{\alpha ; \beta}=\frac{1}{2} \varepsilon \varepsilon_{\alpha \beta}^{\mu v} q_{\mu ; v},
$$

then $g_{\alpha \beta}$ has an $\varepsilon$-self-dual Riemann tensor and admits a local coordinate system in which the metric takes the form:

$$
d s^{2}=V^{-1}(d \tau+\underline{\omega} \cdot \underline{d x})+V d \underline{x}^{2}
$$

with

$$
\underline{\operatorname{curl}} \omega=\varepsilon \underline{\operatorname{grad}} V
$$

Proof. Locally any 4-metric with a Killing vector $\frac{\partial}{\partial \tau}$ may be written as

$$
d s^{2}=V^{-1}(d \tau+\underline{\omega} \cdot \underline{d x})^{2}+V \gamma_{i j} d x^{i} d x^{j} .
$$

The vacuum Einstein equations reduce to the statement that locally

$$
\operatorname{curl} \underline{\omega}=\operatorname{grad} \psi
$$

for some $\psi$, and

$$
R_{i j}=V^{-1}\left(\partial_{i} V \partial_{j} V-\partial_{i} \psi \partial_{j} \psi\right)
$$


The 2-form

$$
d\left(V^{-1}(d \tau+\underline{\omega} \cdot \underline{d x})\right)
$$

is $\varepsilon$-self-dual iff (2.14) holds where the curl and grad operations are covariant with respect to the (a priori curved) metric $\gamma_{i j}$. But (2.1.5) now gives

$$
V=\varepsilon \psi
$$

which results on substitution in (2.1.6) with the result that is Ricci flat, hence (since it is a 3-metric) flat: It now follows that in the obvious basis the connection oneforms are $\varepsilon$-self-dual, and hence that the metric has an $\varepsilon$-self-dual curvature tensor. Given that $g_{\alpha \beta}$ has an $\varepsilon$-self-dual curvature tensor, it follows that $g_{\alpha \beta}$ admits 3 covariantly constant $\varepsilon$-anti-self-dual 2 -forms $F_{\alpha \beta}^{i}$ which may be normalized to satisfy

$$
F_{\alpha}^{i \beta} F_{\beta}^{j}{ }^{\gamma}=-\delta^{i j} \delta_{\alpha}^{\gamma}+\varepsilon^{i j k} F_{\alpha}^{k} .
$$

As explained in [7] $\varepsilon$-duality is equivalent to the existence of a 2 -spheres worth of complex structures with respect to any one of which the metric is Kähler. Such a structure is called in general $4 k$-dimensions a hyperKähler structure.

Since $F_{\alpha \beta}^{i}$ is covariant constant,

$$
{\underset{q}{\alpha}}_{\alpha \beta} F^{i}=F_{\gamma \alpha}^{i} q_{\beta}^{; \gamma}-F_{\gamma \beta}^{i} q_{\alpha}^{; \gamma} .
$$

Because $F_{\alpha \beta}^{i}$ and $q_{\alpha ; \beta}$ have the opposite duality, we obtain

$$
\mathfrak{q}_{q^{\alpha}} F_{\alpha \beta}^{i}=0 \text {. }
$$

Equation (2.1.11) has the significance that the Killing vector is holomorphic with respect to all the complex structures, i.e. the Lie dragging of vectors commutes with multiplication by $i$ in any holomorphic chart. This property is called triholomorphicity. We thus have the following corollary to Theorem (2.1):

Corollary (2.1). Any hyperKähler 4-metric with triholomorphic Killing vector must be of form (2.1.2), (2.1.3).

Corollary (2.1) may be strengthened considerably in view of the following

Proposition (2.2). If a Killing vector $q^{\alpha}$ of a metric with $\varepsilon$-self-dual curvature has an $\varepsilon$-self-dual covariant derivative at a point it has an $\varepsilon$-self-dual covariant derivative everywhere.

Proof. The Ricci identity yields the equation (valid for any Killing vector):

$$
q_{\alpha ; \beta ; \gamma}=R_{\alpha \beta \gamma \lambda} q^{\lambda} \text {. }
$$

This shows (as is well known) that the Killing vector is determined by its value at a single point together with its first covariant derivative. If $q^{\alpha}$ is $\varepsilon$-self-dual, then all the covariant derivatives of $q^{\alpha}$ will be $\varepsilon$-self-dual. Thus if $q^{\alpha}$ is $\varepsilon$-self-dual at a single point it will be $\varepsilon$-self-dual everywhere.

Another useful result is

Theorem 2.3. Let $g_{\alpha \beta}$ be hyperKähler with 2 commuting Killing vectors $q^{\alpha}$ and $p^{\beta}$, then there exists a linear combination which is triholomorphic and hence the metric is of form (2.1.2) and (2.1.3). 
Proof. The Lie bracket of $q^{\alpha}$ and $p^{\beta}$ is

$$
p_{; \beta}^{\alpha} q^{\beta}-q_{; \beta}^{\alpha} p^{\beta} \text {. }
$$

The covariant derivative of (2.1.13) is [using (2.1.12) and the Bianchi Identity] equal to

$$
R_{\alpha \beta \varrho \sigma} p^{\varrho} p^{\sigma}+p_{\alpha ; \gamma} q_{; \beta}^{\gamma}-q_{\alpha ; \gamma} p_{; \beta}^{\gamma} .
$$

If the Riemann tensor is $\varepsilon$-self-dual we may project the vanishing of (2.1.14) on to the space of $-\varepsilon$-self-dual 2 -forms to obtain the result that

$$
\left[P^{-}, Q^{-}\right]=0,
$$

where $P^{-}, Q^{-}$are the $-\varepsilon$-self-dual projections of the 2 -forms $p_{\alpha ; \beta}$ and $q_{\alpha ; \beta}$ and where we are using the obvious matrix multiplication (using the metric). Equation (2.1.15) implies that $P^{-}$and $Q^{-}$are proportional, thus

$$
p_{\alpha ; \beta}+\lambda q_{\alpha ; \beta}
$$

is $\varepsilon$-self-dual for some function $\lambda$. If $\lambda_{p}$ is the value of $\lambda$ at some point $p$ the Killing vector field $K^{\alpha}=p^{\alpha}+\lambda_{p} q^{\alpha}$ has $\varepsilon$-self-dual covariant derivative at $p$ and hence by Proposition (2.2) everywhere.

Remarks. 1) Clearly $V$ must admit an additional symmetry. Typically it will be axisymmetric. It also follows that in the axisymmetric case the "other" Killing vector will leave invariant just one privileged complex structure, i.e. the axisymmetric action is holomorphic with respect to one complex structure, the other two transforming as a doublet of $S O(2)$.

2) We were told of this result by $\mathrm{N}$. Hitchin who has a different proof.

For a metric of form (2.1.2), (2.1.3) the hyperKähler structures are given by [8]

$$
F^{i}=(d \tau+\underline{\omega} \cdot d x) \wedge d x^{i}-V \varepsilon^{i p q} d x^{p} \wedge d x^{q},
$$

which is clearly invariant under $\tau \rightarrow \tau+$ constant. We record here for later convenience the Laplacian in these coordinates [9]:

$$
\nabla_{\alpha} \nabla^{\alpha}=V \frac{\partial^{2}}{\partial \tau^{2}}+V^{-1}\left(\underline{\nabla}-\underline{\omega} \frac{\partial}{\partial \tau}\right)\left(\underline{\nabla}-\underline{\omega} \frac{\partial}{\partial \tau}\right) .
$$

The Green's function and geodesics are given in [9].

To obtain complete metrics we must choose

$$
V=\delta+\sum_{i=1}^{i=N} \frac{2 M}{\left|\underline{x}-\underline{x}_{i}\right|},
$$

where $M>0,0 \leqq \tau \leqq 8 \pi M$, and $\delta=0$ if the metrics are asymptotically locally euclidean (ALE) or $\delta=1$ if they are asymptotically local flat (ALF). Some special cases are

$\delta=0, \quad N=1:$ flat space,

$\delta=1, \quad N=1:$ half flat Taub-NUT (positive mass),

$\delta=0, \quad N=2$ : the Eguchi-Hanson metric,

$\delta=1, \quad N=2:$ the double Taub-NUT metric. 
In connection with monopoles the case $\delta=1, N=1, M<0$ is also of interest. This case is called the half-flat Taub-NUT with negative mass and is Riemannian only if $\left|x-x_{1}\right|>2 M$.

(2.2). The U(2) invariant Taub-NUT family. The following proposition is well known:

Proposition (2.4). The general Ricci flat metric possessing an isometry group acting transitively on 3-dimensional orbits with Lie algebra $U(2)$ has local form:

$$
d s^{2}=\left(1-\frac{2 M t-n^{2}}{t^{2}-n^{2}}\right)^{-1} d t^{2}+4 n^{2}\left(1-\frac{2 M t-n^{2}}{t^{2}-n^{2}}\right) \sigma_{3}^{2}+\left(t^{2}-n^{2}\right)\left(\sigma_{1}^{2}+\sigma_{2}^{2}\right)
$$

where $\sigma_{1}, \sigma_{2}, \sigma_{3}$ are left invariant one-forms on $S U(2)$ with

$$
d \sigma_{1}=\sigma_{2} \wedge \sigma_{3}
$$

and cyclically.

In our conventions $d t \wedge \sigma^{1} \wedge \sigma^{2} \wedge \sigma^{3}$ is positively oriented. The only non-flat half flat $U(2)$ invariant metrics are Eguchi-Hanson and Taub-NUT. The special case $M=|n|$ has self-dual curvature and coincidences with the $\delta=1, N=1$, positive mass half Taub-NUT solutions. The negative mass Taub-NUT solution has $M=-|n|$ and is antiself-dual in our convention. The Eguchi-Hanson metric may be obtained by setting

$$
M=n+a^{2} / 2 n
$$

and taking the limit as $n \rightarrow \infty$.

The $U(2)$ action is both linear and holomorphic in any one of an infinite family of charts defined by

$$
\begin{aligned}
& \zeta^{1}=R(t) \cos \frac{\theta}{2} \exp \frac{i}{2}(\psi+\phi), \\
& \zeta^{2}=R(t) \sin \frac{\theta}{2} \exp \frac{i}{2}(\psi-\phi),
\end{aligned}
$$

where $R(t)$ is an arbitrary function of $t$ and $(\psi, \phi, \theta)$ are the standard Euler angles. Note that two charts in the family are not in general holomorphically related. The metric is Hermitian iff

$$
R^{4}=\left(t-t_{+}\right)^{t_{+} / n}\left(t-t_{-}\right)^{t_{-} / n} \exp (t / n)
$$

or

$$
R^{-4}=\left(t-t_{+}\right)^{t_{+} / n}\left(t-t_{-}\right)^{t_{-} / n} \exp (t / n)
$$

with

$$
t_{ \pm}=M \pm \sqrt{M^{2}-n^{2}}
$$

Since Ricci flat Kähler implies hyperKähler in 4-dimensions, the only possible non-trivial cases in which this Hermitian structure could also be Kähler are the Taub-NUT or Eguchi-Hanson cases. By examination one finds that this occurs only in the latter case. Thus: 
Proposition (2.5). The only hyperKähler 4-metric with linear and holomorphic U(2) action is the Eguchi-Hanson metric with holomorphic coordinates defined by

$$
R^{4}=a^{2}\left(\varrho^{2}-a^{2}\right) .
$$

This proposition is derived directly in [12] together with the Kähler form.

In fact, one can say something stronger in view of the

Proposition (2.6). Given any hyperKähler 4-metric with an $S U(2)$ action which is holomorphic with respect to one of the complex structures, then the action is necessarily triholomorphic.

Proof. The complex structures carry a representation of any subgroup of the isometry group of the metric. Hence in this case $S U(2)$ has a real 3-dimensional representation on the complex structures. This is either the triplet or 3 singlet representations. Since one complex structure is invariant under $S U(2)$, the representation cannot be irreducible and hence all complex structures are invariant.

Thus in Eguchi-Hanson we have

$$
\underset{J^{i}}{\mathfrak{f}} F_{\alpha \beta}^{k}=0
$$

where $J^{i}$ generate the $S O(3)$ subgroup. In Eguchi-Hanson the $U(1)$ factor of $U(2)$ does not act holomorphically with respect to all 3 complex structures. One of them [given by (2.2.8)] is invariant but the orthogonal pair rotate as a real doublet of $U(1)$. An example of a Kähler chart for which the $S U(2)$ is holomorphic but the $U(1)$ is not and its relation to the privileged complex structure (2.2.8) is given in $[10]$.

The situation should be contrasted with that of Taub-NUT for which the $S O(3)$ is not holomorphic but rather [by Proposition (2.6)] must satisfy

$$
\underset{J^{i}}{£} F_{\alpha \beta}^{k}=\varepsilon^{i k \ell} F_{\alpha \beta}^{\ell} \text {. }
$$

In fact, Eq. (2.2.9) is obvious from the explicit form (2.1.16). For Taub-NUT the most convenient choice for the arbitrary function $R(t)$ in (2.2.3), (2.2.4) is $\sqrt{t-n} \equiv \sqrt{r}$. These coordinates will be denoted by $Z^{1}$ and $Z^{2}$ in what follows. The Kähler coordinates for Taub-NUT have been given in [11]. In our conventions we define (using standard polar coordinates for $\mathbb{R}^{3}$ )

$$
\begin{gathered}
w=r \sin \theta e^{i \phi}=x+i y, \\
v=r \cos \frac{\theta}{2} \exp \left(\frac{4 r}{M} \cos \theta+\frac{i \tau}{4 M}\right),
\end{gathered}
$$

whence the metric (2.1.2) with $V=1+2 M / r$ takes the manifestly Hermitian form:

$$
d s^{2}=\left(1+\frac{2 M}{r}\right)^{-1}|d w|^{2}+\left(1+\frac{2 M}{r}\right)\left|\frac{d v}{v}-\sin ^{2} \frac{\theta}{2} \frac{d w}{w}\right|^{2} .
$$

One may verify that the fundamental 2 -form is closed and hence Kähler. Evidently the choice of the $z$ direction is arbitrary up to the action of $S O(3)$ and so there 
indeed exists a 2-sphere's worth of complex structures defined in this way. The relation of the multi-centre form of Eguchi-Hanson to the manifestly $U(2)$ invariant form (2.2.1) will be described later.

The $U(2)$ invariant metrics have Petrov type $D^{+}, D^{-}$. To see this recall that we may regard the Weyl tensor $C_{\alpha \beta}{ }^{\mu \nu}$ as a linear map from 2-forms to 2-forms with vanishing trace. The symmetries of the Weyl tensor imply that the map is symmetric with respect to the standard induced metric on 2-forms and that it commutes with Hodge dual. Thus we obtain 2 real $3 \times 3$ symmetric matrices with vanishing trace. Then either is said to be of type $D$, if two of the eigenvalues are equal. The matrices are invariant under the induced orthogonal action of the isotropy subgroup of the isometry group. In the Taub-NUT family the isotropy subgroup is the $U(1)$ factor of $U(2)$. The $U(1)$ can thus only rotate 2 eigendirections with equal eigenvalue about the third eigendirection, and so both matrices must be of type $D$ (of course, one vanishes in the half flat case).

For the record we note that the entire family admits a $U(2)$ invariant KillingYano 2-form $Y$ satisfying

$$
\frac{1}{2} d Y=\nabla Y
$$

and given by:

$$
Y=t\left(t^{2}-n^{2}\right) \sin \theta d \theta \wedge d \phi+2 n^{2} d t \wedge(d \psi+\cos \theta d \phi) .
$$

In the Eguchi-Hanson limit $Y$ coincides (after rescaling) with the privileged $U(1)$ invariant complex structure.

(2.3). The $S O(3)$ invariant half-flat metrics. The requirement that a half-flat 4-metric admit an $S O(3)$ action with (generally) 3-dimensional orbits leads to ordinary differential equations [12] which were completely investigated in [3] and also [13]. Their results may be summarized by the following

Proposition (2.7). The only SO(3) complete non-singular invariant half-flat 4-metrics with 3-dimensional orbits are

1) the Atiyah-Hitchin metric;

2) the Taub-NUT metric with positive mass;

3) the Eguchi-Hanson metric.

Of these only the Atiyah-Hitchin metric does not admit a further U(1). In AtiyahHitchin as in Taub-NUT the SO(3) action is holomorphic with respect to none of the Kähler structures. The Atiyah-Hitchin metric cannot therefore be cast in the form (2.1.2), (2.1.3), i.e. it does not admit a triholomorphic U(1) action.

The asymptotic form of the Atiyah-Hitchin metric is that of the Taub-NUT metric with a negative mass [3].

The complex structures in Eguchi-Hanson may be written as:

$$
\omega^{0} \wedge \omega^{i}+\frac{1}{2} \varepsilon^{i j k} \omega^{j} \wedge \omega^{k}
$$

where the $\omega^{i}$ are orthonormal 1 -forms parallel to the $\sigma_{i}$ and $\omega^{0}$ is the 4 th leg of the orthonormal frame. The privileged complex structure is:

$$
\omega^{0} \wedge \omega^{3}+\omega^{1} \wedge \omega^{2}
$$




\section{The Conserved Quantities and Killing Tensors}

The geodesic Lagrangian in negative mass Taub-NUT is

$$
L=\pi\left[\left(1-\frac{2}{r}\right) \underline{\dot{r}} \cdot \dot{\dot{r}}+4\left(1-\frac{2}{r}\right)(\dot{\psi}+\cos \theta \dot{\phi})^{2}\right] .
$$

The conserved momentum conjugate to the ignorable coordinate $\psi$ is

$$
q=8 \pi\left(1-\frac{2}{r}\right)^{-1}(\dot{\psi}+\cos \theta \dot{\phi})
$$

and the conserved energy $E$ is

$$
E=\pi\left(1-\frac{2}{r}\right)\left(\underline{r}^{2}+\left(\frac{q}{4 \pi}\right)^{2}\right) .
$$

The conserved angular momenta $\underline{J}$ are:

$$
\underline{J}=2 \pi\left(1-\frac{2}{r}\right) \underline{r} \times \underline{\dot{r}}-q \underline{\hat{r}},
$$

where $\underline{\hat{r}}$ is a unit vector in the $\underline{r}=\underline{x}-\underline{x}_{1}$ direction. In addition in [5] it was shown that there are 3 additional conserved quantities $\underline{K}$, defined by

$$
\underline{K}=2 \pi\left(1-\frac{2}{r}\right) \underline{\dot{r}} \times \underline{J}+\left(4 \pi E-\frac{1}{2} q^{2}\right) \underline{\hat{r}} .
$$

The vector $\underline{K}$ generalizes the Runge-Lenz vector of the standard Coulomb problem. The non-vanishing Poisson brackets are (after some algebra) (see also [14]),

$$
\begin{gathered}
\left\{J^{i}, J^{j}\right\}=\varepsilon^{i j k} J^{k}, \\
\left\{J^{i}, K^{j}\right\}=\varepsilon^{i j k} K^{k}, \\
\left\{K^{i}, K^{j}\right\}=\left(\frac{q^{2}}{4}-4 \pi E\right) \varepsilon^{i j k} J^{k} .
\end{gathered}
$$

The sign of the quantity $\left(\frac{q^{2}}{4}-4 \pi E\right)$ distinguishes between bound and unbound states being positive in the former and negative in the latter. The algebra may be put in a more recognizable form by defining:

$$
\begin{gathered}
\underline{M}=\left|\frac{q^{2}}{4}-4 \pi E\right|^{-1 / 2} \underline{K}, \\
\underline{A}_{ \pm}=\frac{1}{2}(\underline{J} \pm \underline{M}),
\end{gathered}
$$

whence (for bound states)

$$
\begin{gathered}
\left\{A^{i}{ }_{ \pm}, A^{j}{ }_{ \pm}\right\}=\varepsilon^{i j k} A^{k}{ }_{ \pm}, \\
\left\{A^{i}{ }_{+}, A^{j}{ }_{-}\right\}=0 .
\end{gathered}
$$


This is the Lie algebra of $s u(2) \oplus s u(2)$. The magnitudes of the vectors $\underline{A}_{ \pm}$are given by:

$$
\underline{A}^{2}{ }_{ \pm}=\frac{1}{4}\left(q \pm \frac{\left(\frac{q^{2}}{2}-4 \pi E\right)}{\left(\frac{q^{2}}{4}-4 \pi E\right)^{1 / 2}}\right)^{2}=\frac{1}{4} \frac{\left(\frac{q}{2} \pm\left(\frac{q^{2}}{4}-4 \pi E\right)^{1 / 2}\right)^{4}}{\left(\frac{q^{2}}{4}-4 \pi E\right)} .
$$

At fixed $E$ and $q$ the space of classical bound orbits is specified by the 2 constants $\underline{A}_{ \pm}$subject to (3.13). This means that the classical bound orbits are in $1-1$ correspondence with the points of $S^{2} \times S^{2}$. From (3.11), (3.12) it follows that the canonical transformations generated by $\underline{A}_{ \pm}$act multiply transitively on this space $\underline{A}_{+}$acting on one factor and $\underline{A}_{-}$on the other.

The functions $J$ and $q$ are linear in 4-velocities and arise from the Killing vectors of this metric in the standard way:

$$
\begin{aligned}
q & =q_{\alpha} \frac{d x^{\alpha}}{d t}, \\
J^{i} & =\xi_{L}^{i} \frac{d x^{\alpha}}{d t} .
\end{aligned}
$$

The functions $\underline{K}$ are quadratic in 4-velocities and may thus be written as

$$
K^{i}=K_{\alpha \beta}^{i} \frac{d x^{\alpha}}{d t} \frac{d x^{\beta}}{d t}
$$

where the $K_{\alpha \beta}^{i}=K_{\beta \alpha}^{i}$ are 3 Killing tensors which by definition satisfy

$$
K_{(\alpha \beta ; \gamma)}^{i}=0 .
$$

Equation (3.17) is the necessary and sufficient condition that the right-hand side of (3.16) is constant along geodesics. The necessary and sufficient condition that the second order formally self-adjoint operators

$$
\hat{K}^{i} \equiv \nabla^{\alpha}\left(K_{\alpha \beta}^{i} \nabla^{\beta}\right)
$$

commute with the D'Alembertian [15],

$$
\left(K_{\gamma}^{i}{ }^{[\alpha} R^{\beta] \gamma}\right)_{; \beta}=0,
$$

is also satisfied in the Taub-NUT metric by virtue of the vanishing of the Ricci tensor.

One may check by explicit calculation the following

Proposition 3.1. The Killing tensors $K_{\alpha \beta}^{i}$ are related to the $U(2)$ invariant KillingYano tensor $Y_{\alpha \beta}$ and the complex structures $F_{\alpha \beta}^{i}$ by

$$
K_{\alpha \beta}^{i}=Y_{\gamma(\alpha} F_{\beta)}^{i \gamma} \text {. }
$$


In fact, explicitly one has:

$$
\begin{gathered}
Y=\omega^{0} \wedge \omega^{3}+(r-1) \omega^{1} \wedge \omega^{2} \\
F^{1}=2(d \psi+\cos \theta d \phi) \wedge d x+\left(1-\frac{2}{r}\right) d y \wedge d z \\
F^{2}=2(d \psi+\cos \theta d \phi) \wedge d y+\left(1-\frac{2}{r}\right) d z \wedge d x \\
F^{3}=2(d \psi+\cos \theta d \phi) \wedge d z+\left(1-\frac{2}{r}\right) d x \wedge d y,
\end{gathered}
$$

where $\omega^{0}, \omega^{1}, \omega^{2}, \omega^{3}$ is the orthonormal basis given by Eq. (2.2.1), $r=t+1$ and we have taken $M=-|n|=-1$. The constant of the motion obtained by squaring $Y$, i.e.

$$
K_{\alpha \beta}=Y_{\alpha}^{\sigma} Y_{\sigma \beta}
$$

is not new, in fact,

$$
K_{\alpha \beta} \frac{d x^{\alpha}}{d t} \frac{d x^{\beta}}{d t}=\frac{\underline{J}^{2}-q^{2}}{(4 \pi)^{2}}+\frac{E}{\pi}
$$

It is clear from (3.21) that $Y_{\alpha \beta}$ is both $U(1)$ and $S O(3)$ invariant, i.e.

$$
\begin{aligned}
& \underset{q^{\mu}}{£} Y_{\alpha \beta}=0, \\
& \underset{\xi_{L}^{\prime \alpha}}{\mathfrak{f}} Y_{\alpha \beta}=0 .
\end{aligned}
$$

In the next section we shall relate the existence of the Killing tensors to separation of variables of the Hamilton-Jacobi and Schrödinger equations. Then in the following section we shall discuss the implications of the existence of a YanoKilling tensor.

\section{Separability and Killing Tensors}

Killing tensors often arise as separation constants for the Hamiltonian-Jacobi and/or Schrödinger equations. Rather than describing this connection in general (see e.g. $[16,17]$ ) we shall limit ourselves to giving a detailed discussion of a particular class of " 2 centre" metrics which include as special cases the Taub-NUT, Eguchi-Hanson, and double Taub-NUT. The separability occurs in spheroidal coordinates adapted to the 2 centres and generalizes the well known fact in atomic physics [18] that the Schrödinger equation for a diatomic molecule separates in much the same way that the extra conserved quantities in the Taub-NUT metric generalize the existence of the Runge-Lenz vector in the Hydrogen atom problem.

We begin by defining spheroidal coordinates $(\zeta, \lambda, \sigma)$ based on two points $\underline{x}=\underline{x}_{1}$ and $\underline{x}=\underline{x}_{2}$ in $\mathbb{R}^{3}$. Define the distances from the two points by

$$
r_{1}=\left|\underline{x}-\underline{x}_{1}\right| ; \quad r_{2}=\left|\underline{x}-\underline{x}_{2}\right| .
$$

Fix a plane passing through $\underline{x}_{1}$ and $\underline{x}_{2}$. A point not in the line through $\underline{x}_{1}$ and $\underline{x}_{2}$ lies in a unique plane intersecting that line and making an angle $\sigma$ with it. The 
angles between line $x_{1} x_{2}$ and the lines $x_{1} x$ and $x_{2} x$ are $\theta_{1}$ and $\theta_{2}$. Then $\zeta, \lambda$ are defined by

$$
r_{1}+r_{2}=2 R \zeta, \quad r_{1}-r_{2}=2 R \lambda,
$$

where $2 R=\left|\underline{x}_{1}-\underline{x}_{2}\right|$. Thus:

$$
\cos \theta_{1}=\frac{\zeta \lambda+1}{\zeta+\lambda}, \quad \cos \theta_{2}=\frac{\zeta \lambda-1}{\zeta+\lambda},
$$

and the Euclidean metric on $\mathbb{R}^{3}$ takes the form

$$
d s^{2}=R^{2}\left(\zeta^{2}-\lambda^{2}\right)\left(\frac{d \zeta^{2}}{\zeta^{2}-1}+\frac{d \lambda^{2}}{1-\lambda^{2}}\right)+R^{2}\left(\zeta^{2}-1\right)\left(1-\lambda^{2}\right) d \sigma^{2} .
$$

If one point, say $\underline{x}_{2}$, is allowed to recede to infinity we obtain the parabolic coordinates used in [5] where our $\sigma$ is the $\phi$ of [5], i.e.

$$
\eta=\operatorname{Lt}_{R \rightarrow \infty} 2 R(\zeta-1) ; \quad \xi=\operatorname{Lt}_{R \rightarrow \infty} 2 R(\lambda+1) .
$$

We shall consider the general metric

$$
d s^{2}=F^{-1} 4 R^{2}\left(d \chi^{2}+\left(\alpha \cos \theta_{1}+\beta \cos \theta_{2}\right) d \sigma\right)^{2}+F d \underline{x}^{2},
$$

where

$$
F=\left(K+\frac{R \gamma}{r_{1}}+\frac{R \delta}{r_{2}}\right)
$$

The metric (4.6) may be thought of as a combination of 2 gravitational dyons. Consider the equation

$$
-\nabla^{2} f=\left(\varepsilon+\frac{\omega^{2}}{F}\right) f
$$

where $\omega^{2} / F$ may be regarded as a potential term. Equation (4.8) becomes:

$$
\begin{aligned}
\frac{\partial}{\partial \zeta}\left(\left(\zeta^{2}-1\right) \frac{\partial f}{\partial \zeta}\right)+\frac{\partial}{\partial \lambda}\left(\left(1-\lambda^{2}\right) \frac{\partial f}{\partial \lambda}\right)+\left(\frac{1}{\zeta^{2}-1}+\frac{1}{1-\lambda^{2}}\right) \\
\quad \times\left(\frac{\partial}{\partial \sigma}-\alpha \cdot \frac{\lambda\left(\zeta^{2}-1\right)+\zeta\left(1-\lambda^{2}\right)}{\zeta^{2}-\lambda^{2}} \frac{\partial}{\partial \chi}-\beta \cdot \frac{\lambda\left(\zeta^{2}-1\right)-\zeta\left(1-\lambda^{2}\right)}{\zeta^{2}-\lambda^{2}} \frac{\partial}{\partial \chi}\right)^{2} f \\
\quad+\frac{1}{4} \frac{1}{\zeta^{2}-\lambda^{2}}\left(K\left(\zeta^{2}-\lambda^{2}\right)+\gamma(\zeta-\lambda)+\delta(\zeta+\lambda)\right)^{2} \frac{\partial^{2} f}{\partial x^{2}} \\
=\varepsilon R^{2}\left(K\left(\zeta^{2}-\lambda^{2}\right)+\gamma(\zeta-\lambda)+\delta(\zeta+\lambda)\right) f+\omega^{2} R^{2} f
\end{aligned}
$$

The coefficient of $\frac{\partial^{2} f}{\partial \sigma \partial \chi}$ is

$$
-\frac{2 \lambda(\alpha+\beta)}{1-\lambda^{2}}-\frac{2 \zeta(\alpha-\beta)}{\zeta^{2}-1}
$$


That of $\frac{\partial^{2} f}{\partial \chi^{2}}$ is

$$
\begin{aligned}
& \frac{1}{4} K^{2}\left(\zeta^{2}-\lambda^{2}\right)+\frac{1}{2} \gamma K(\zeta-\lambda)+\frac{1}{2} K \delta(\zeta+\lambda)+\frac{1}{4} \frac{\gamma^{2}(\zeta-\lambda)^{2}+\delta^{2}(\zeta+\lambda)^{2}}{\zeta^{2}-\lambda^{2}}+\frac{1}{2} \gamma \delta \\
& \quad+(\alpha+\beta)^{2} \frac{\lambda^{2}}{1-\lambda^{2}}+(\alpha-\beta)^{2} \frac{\zeta^{2}}{\zeta^{2}-1}+\frac{1}{\zeta^{2}-\lambda^{2}}\left(2\left(\alpha^{2}-\beta^{2}\right) \lambda \zeta\right. \\
& \left.\quad-(\alpha+\beta)^{2} \lambda^{2}-(\alpha-\beta)^{2} \zeta^{2}\right) .
\end{aligned}
$$

Separability clearly occurs if the numerator of $\frac{1}{\zeta^{2}-x^{2}}$ contains a factor of $\zeta^{2}-\lambda^{2}$. Since the numerator is quadratic in $\zeta, \lambda$ it must in fact be a multiple of $\zeta^{2}-\lambda^{2}$. Thus we have the following

Proposition (4.1). The sufficient conditions for Eq. (4.8) to be multiplicatively separable are:

$$
\gamma^{2}=4 \alpha^{2}, \quad \delta^{2}=4 \beta^{2} .
$$

Equations (4.12) imply that the 2 dyons are separately either self-dual or antiself-dual. Thus condition (4.12) is analogous to the cancellation required in the Taub-NUT metric to allow the existence of extra conserved quantities, the term $\omega^{2} F$ being the analogue of the term arising in the generalization described in [6]. The metric (4.6) subject to (4.12) is Ricci flat iff $\alpha \beta \gamma \delta>0$.

Proposition (4.1) contains two already known cases:

I) If one of the points recedes to infinity, $R \rightarrow \infty$, we obtain the separability in parabolic coordinates for the Taub-NUT metric and Taub-NUT with potential term noted in $[5,6]$.

II) If $K=0=\omega^{2}$ and $\alpha=\beta$, we obtain a result which follows from the fact that Eguchi-Hanson is in fact $U(2)$ invariant and Proposition (4.1) amounts to separation of variables in radial coordinates. We shall explain this further later.

An interesting new result is that if $\delta=0$ we obtain separability in Taub-NUT (with or without potential term) in a new set of coordinates. In the related Coulomb case this allows a fairly explicit formula for the Greens function [19-21] and in the present case leads to a simple explicit formula for the $\psi$ averaged Greens function which is easily deduced from that given in [21]. If one averages over $\psi$ the magnetic term in (2.1.17) does not contribute. Unfortunately, the method of [21] doesn't readily generalize to the case where magnetic terms are present. If $\varepsilon=\omega^{2}=0$, one has an explicit formula for the Greens function due to Page [9].

It follows from Proposition (4.1) and the semi-classical expansion that the Hamilton-Jacobi equation for the metric (4.6) is additively separable. The Hamilton-Jacobi equation is

$$
\begin{aligned}
\left(\zeta^{2}-1\right) & \left(\frac{\partial S}{\partial \zeta}\right)^{2}+\left(1-\lambda^{2}\right)\left(\frac{\partial S}{\partial \lambda}\right)^{2}+\left(\frac{1}{\zeta^{2}-1}+\frac{1}{1-\lambda^{2}}\right) \\
& \times\left(\frac{\partial S}{\partial \sigma}-\frac{(\alpha+\beta) \lambda\left(\zeta^{2}-1\right)+(\alpha-\beta) \zeta\left(1-\lambda^{2}\right)}{\partial \chi}\right)^{2} \\
& +\frac{1}{4} \frac{1}{\zeta^{2}-\lambda^{2}}\left(K\left(\zeta^{2}-\lambda^{2}\right)+\gamma(\zeta-\lambda)+\delta(\zeta+\lambda)\right)^{2}\left(\frac{\partial S}{\partial \chi}\right)^{2} \\
= & \varepsilon R^{2}\left(K\left(\zeta^{2}-\lambda^{2}\right)+\gamma(\zeta-\lambda)+\delta(\zeta+\lambda)\right)+\omega^{2} R^{2} .
\end{aligned}
$$


The last term in (4.13) is absent if one is merely considering geodesics. If

$$
S=\chi P_{\chi}+\sigma P_{\sigma}+W(\zeta)+U(\lambda),
$$

where $P_{\chi}$ and $P_{\sigma}$ are the constant momenta conjugate to $x$ and $\sigma$ respectively and where the momenta conjugate to $\zeta$, respectively $\lambda$ are

$$
P_{\zeta}=\frac{d W}{d \zeta} ; \quad P_{\lambda}=\frac{d U}{d \lambda}
$$

then we can write (4.13) as the 2 separated equations:

$$
\begin{aligned}
& \left(1-\lambda^{2}\right) P_{\lambda}^{2}+\frac{1}{1-\lambda^{2}} P_{\sigma}^{2}-\frac{2 \lambda(\alpha+\beta)}{1-\lambda^{2}} P_{\chi} P_{\sigma}+\left(\frac{1}{2} K \delta \lambda-\frac{1}{4} K^{2} \lambda^{2}-\frac{1}{2} \gamma K \lambda\right) P_{\chi}^{2} \\
& =\varepsilon R^{2}\left(\delta \lambda-\gamma \lambda-K \lambda^{2}\right)+C_{\lambda},
\end{aligned}
$$

and

$$
\begin{aligned}
\left(\zeta^{2}-1\right) P_{\zeta}^{2}+\frac{1}{\zeta^{2}-1} P_{\sigma}^{2}-\frac{2 \zeta(\alpha-\beta)}{\zeta^{2}-1} P_{\sigma} P_{x} \\
\quad+\left(\frac{1}{4} K^{2} \zeta^{2}+\frac{K}{2}(\gamma+\delta) \zeta+\frac{1}{2} \gamma \delta+2 \alpha \beta\right) P_{x}^{2} \\
=\varepsilon R^{2}\left(K \zeta^{2}+\zeta(\gamma+\delta)\right)+C_{\zeta},
\end{aligned}
$$

where $C_{\lambda}, C_{\zeta}$ are separation constants such that

$$
C_{\zeta}+C_{\lambda}=\omega^{2} R^{2}
$$

Since

$$
\frac{1}{2} g^{\alpha \beta} P_{\alpha} P_{\beta}=\varepsilon+\omega^{2} / F,
$$

we may substitute (4.19) into (4.16) and (4.18) to express $C_{\lambda}$ and $C_{\zeta}$ as the sum of 2 terms, one quadratic in momenta and one independent of momenta. Thus

$$
\begin{aligned}
& C_{\zeta}=C_{\zeta}{ }^{\alpha \beta} P_{\alpha} P_{\beta}+\omega^{2} Q_{\zeta}, \\
& C_{\lambda}=C_{\lambda}^{\alpha \beta} P_{\alpha} P_{\beta}+\omega^{2} Q_{\lambda} .
\end{aligned}
$$

The equations of motion may be written as

$$
\dot{x}_{; \beta}^{\alpha} \dot{x}^{\beta}=\omega^{2} g^{\alpha \beta} \partial_{\beta}\left(\frac{1}{F}\right)=P_{; \beta}^{\alpha} P^{\beta} .
$$

The constancy of $C_{\zeta}$ and $C_{\lambda}$ requires that:

$$
C_{\zeta \alpha \beta ; \gamma} P^{\alpha} P^{\beta} P^{\gamma}+2 C_{\zeta}^{2 \beta} P_{\alpha} \omega^{2} \partial_{\beta}\left(\frac{1}{F}\right)+\omega^{2} Q_{\zeta, \alpha} P^{\alpha}=0,
$$

with a similar equation for $C_{\lambda}$. that

Equation (4.24) must be true for all $\omega^{2}$ and all initial momenta $P^{\alpha}$. It follows

$$
C_{\zeta(\alpha \beta ; \gamma)}=-C_{\lambda(\alpha \beta ; \gamma)}=0,
$$


and

$$
2 C_{\zeta \alpha \beta} \nabla^{\beta}\left(\frac{1}{F}\right)+Q_{\zeta, \alpha}=0,
$$

and similarly $C_{\lambda \alpha \beta}$ and $Q_{\lambda}=-Q_{\zeta}+R^{2}$.

Equation (4.24) tells us that the metric (4.6) admits a quadratic Killing tensor.

The existence of 4 Poisson commuting constants of the motion shows

Proposition (4.2). The dynamical system with Hamiltonian

$$
H=\frac{1}{2} g^{\alpha \beta} P_{\alpha} P_{\beta}-\omega^{2} / F
$$

is integrable in the Liouville sense.

$\partial$ Thus in addition to the symmetries corresponding to the Killing vectors $\frac{\partial}{\partial \chi}$ and $\frac{\partial}{\partial \sigma}$ there are further symmetries corresponding to translating the remaining (action) angle variables. Even in the molecular physics applications (i.e. $P_{x}=0$ ) the symmetries of the 2 centre problem are not very well understood (see $[22,23]$ ) so we shall pass here to the 2 special cases I) Eguchi-Hanson, $K=0=\omega^{2}, \gamma=2 \alpha$ $=\delta=2 \beta$ and II) Taub-NUT, $K=1, \omega^{2}=0, \gamma=2 \alpha, \delta=0=\beta$.

I) Eguchi-Hanson. Using the freedom to set $\gamma=2, \alpha=1$ we allow $\sigma$ and $x$ the ranges $0 \leqq \sigma \leqq 2 \pi ; 0 \leqq x \leqq 4 \pi$. This metric is well known to admit a $U(2)$ isometry group which is not apparent from the metric form (4.6) which has only the maximal commuting subgroup $U(1) \times U(1)$ manifest. To obtain the standard form define $\theta$, $\varrho, \phi$, and $\psi$ by

$$
\begin{aligned}
2 R \cos \theta & =r_{1}-r_{2}, \\
\frac{\varrho^{2}}{4} & =r_{1}+r_{2}, \\
\phi & =\frac{1}{2} x, \\
\psi & =\sigma,
\end{aligned}
$$

to cast the metric in the form:

$$
\begin{aligned}
d s^{2}= & \frac{\varrho^{2}}{2}\left(1-\frac{64 R^{2}}{\varrho^{4}}\right)(d \psi+\cos \theta d \phi)^{2}+\left(1-\frac{64 R^{2}}{\varrho^{4}}\right)^{-1} \frac{d \varrho^{2}}{8} \\
& +\frac{\varrho^{2}}{2}\left(d \theta^{2}+\sin ^{2} \theta d \phi^{2}\right) .
\end{aligned}
$$

The constant $C_{\lambda \alpha \beta} P^{\alpha} P^{\beta}$ becomes:

$$
C_{\lambda}^{\alpha \beta} P_{\alpha} P_{\beta}=\left\{P_{\theta}^{2}+\frac{1}{\sin ^{2} \theta}\left(P_{\psi}-\cos \theta P_{\phi}\right)^{2}+\frac{P_{\phi}^{2}}{\sin ^{2} \theta}\right\}-P_{\phi}^{2} .
$$

Equation (4.29) shows that the Killing tensor $C_{\lambda \alpha \beta}$ is reducible in this case since the term in braces in (4.29) is just the total angular momentum and the second its 
conserved third component squared. For the reader's convenience we point out that the Killing vector $\frac{\partial}{\partial \phi}$ is triholomorphic while $\frac{\partial}{\partial \psi}$ is merely holomorphic with respect to the preferred complex structure. Despite appearances in order to cast (4.28) in multi-centre form one is obliged to follow Eq. (4.27) and identify the Euler angle $\psi$ with the azimuthal angle $\sigma$ in the multi-centre representation (4.6). This is clearly necessary since $\frac{\partial}{\partial \sigma}$ is not triholomorphic (see after Proposition 2.6).

II) Taub-NUT. In distinction to the Eguchi-Hanson case the Killing tensor $C_{\lambda}{ }^{\alpha \beta}$ is really a new constant of the motion in this case. Since the position, $\underline{x}_{2}$, of the second mass point and the plane through the points $\underline{x}_{2}$ and $\underline{x}_{1}$ are now arbitrary, it follows that we obtain in this way a 4 parameter family of constants which may be obtained from one another by scaling and rotations. For fixed scale, i.e. fixed $R$, they transform as a vector under $S O(3)$. Since the space of orbits (at fixed energy and electric charge) is 4-dimensional, each orbit being specified by two vectors $\underline{A}_{ \pm}$ subject to (3.13), these new constants of the motion must be functions of the already obtained, $\underline{A}_{ \pm}$. If we allow the point $\underline{x}_{2}$ to recede to infinity along a fixed line through $\underline{x}_{2}$ the spheroidal coordinates become parabolic coordinates as mentioned above. The separation constant $C_{\lambda}{ }^{\alpha \beta} P_{\alpha} P_{\beta}$ then reduces to the separation constant obtained in parabolic coordinates. In the closely related Hydrogen atom problem it is known [24] that this parabolic separation constant coincides with one component of the Runge-Lenz vector. It seems plausible, but we have not checked this in detail, that a similar relation should hold in our case.

To conclude this section we remark that it seems to be widely believed that a Ricci flat 4-metric admitting two commuting Killing vectors and allowing separation of variables for the Schrödinger equation must be of Petrov type $D$. We have computed the Petrov type of the metrics (4.6) in the particular 2-centre case with $K \neq 0, \alpha=\beta, \gamma=\delta$. It is not Petrov type $D$.

\section{Killing-Yano Tensors}

A second rank Killing-Yano tensor or Yano tensor for short satisfies by definition [25]

$$
Y_{\alpha \beta ; \gamma}=Y_{[\alpha \beta ; \gamma]} \text {. }
$$

They arise in a natural way if one has a problem in which a spin vector $S^{\alpha}$ is covariantly constant along a geodesic with momentum $P^{\alpha}$. Given the existence of a Yano tensor one obtains spin vectors satisfying this condition by the formula:

$$
S^{\alpha}=Y_{\beta}^{\alpha} P^{\beta} \text {. }
$$

One can view Yano tensors as being in some sense the square roots of Killing tensors by virtue of the easily verified

Proposition 5.1. The symmetrized product,

$$
Y_{(\alpha}^{1}{ }^{\sigma}{ }_{|\sigma| \beta)}^{2},
$$

of 2 Yano tensors $Y_{\alpha \beta}^{1}$ and $Y_{\alpha \beta}^{2}$ is a Killing tensor. 
In the $U(2)$ invariant Taub-NUT geometry the square of the Killing tensor $Y_{\alpha \beta}$ given by (2.2.11) gives rise to the reducible constant of the motion:

$$
\frac{n^{2} E}{\pi}+\frac{1}{4 \pi^{2}}\left(J^{2}-q^{2}\right)
$$

where $E$ is the energy. To obtain irreducible Killing tensors using Proposition 5.1 we may take the products of Kähler structures (which trivially satisfy 5.1) with $Y_{\alpha \beta}$. As mentioned in Sect. 3, Eq. (3.20) this will indeed produce the Killing tensors $K_{\alpha \beta}^{i}$ and [by virtue of the relations (2.1.9)] these are the only non-trivial Killing vectors we can obtain in this way. That $Y_{\alpha \beta}$ has no particular duality in the $U(2)$ family except in the Eguchi-Hanson case where $Y^{\alpha}{ }_{\beta}$ coincides with the privileged complex structure may be understood from the following

Proposition 5.2. A Yano tensor $Y_{\alpha \beta}$ has a fixed duality iff it is proportional to the Kähler form of a Kähler metric.

Proof. The definition (5.1) is equivalent to

$$
\frac{1}{2} d Y=\nabla Y \text {. }
$$

Contraction on $\beta$ and $\gamma$ in (5.1) implies

$$
\delta Y=0 .
$$

If $Y$ has a fixed duality (5.6) implies that

$$
d Y=0,
$$

and hence by (5.5) that $Y$ is covariantly constant.

Since for any 2-form

$$
Y^{\alpha}{ }_{\beta} * Y^{\beta}{ }_{\gamma}=-\frac{1}{4}\left(Y_{\varepsilon \sigma} * Y^{\varepsilon \sigma}\right) \delta^{\alpha}{ }_{\gamma},
$$

$Y_{\beta}^{\alpha}$ must have maximal rank and by dividing $Y^{\alpha}{ }_{\beta}$ by the constant nonvanishing number $\left(\frac{1}{4} Y_{\varepsilon \sigma} * Y^{\varepsilon \sigma}\right)^{1 / 2}$ we can normalize $Y^{\alpha}{ }_{\beta}$ to satisfy

$$
Y_{\beta}^{\alpha} Y_{\gamma}^{\beta}=-\delta_{\gamma}^{\alpha} \text {. }
$$

Thus $Y_{\beta}^{\alpha}$ satisfies the necessary and sufficient conditions to be the complex structure of a Kähler metric. Note that if the metric is Ricci flat then it must further be hyperKähler.

If, however, $Y_{\alpha \beta}$ does not have definite duality one can define the following nonzero vector field

$$
K^{\mu}=\frac{1}{3 !} \varepsilon^{\mu v \varrho \sigma} Y_{\gamma \varrho ; \sigma} .
$$

The vector field $K^{\mu}$ is called the primary Killing vector field associated to the Yano tensor $Y_{\alpha \beta}$ [26]. This name is justified by virtue of the

Proposition 5.3. The vector field $K^{\mu}$ is Killing if $Y_{\alpha \beta}$ has maximal rank or the metric is Ricci flat.

Proof. We first establish the following two necessary conditions that $Y_{\mu v}$ must satisfy [15]

$$
Y_{\mu v ; \varrho ; \sigma}=\frac{3}{2} Y_{\tau[v} R_{\mu \varrho] \sigma}^{\tau}
$$


and

$$
R_{\mu v[\sigma}{ }^{\tau} Y_{\varrho] \tau}+R_{\sigma \varrho[\mu}{ }^{\tau} Y_{v] \tau}=0
$$

We have from (5.1)

$$
Y_{\alpha \beta ; \gamma}+Y_{\alpha \gamma ; \beta}=0 \text {. }
$$

Now taking the covariant derivative of $Y_{\alpha \beta ; \gamma}$ and successively using the Ricci identity and (5.13) one obtains:

$$
2 Y_{\mu \nu ; \lambda ; \varrho}=R_{\mu \sigma \nu \varrho} Y_{\lambda}^{\sigma}+R_{\lambda \sigma \nu \varrho} Y_{\mu}^{\sigma}+R_{\mu \sigma \nu \lambda} Y^{\sigma}{ }_{\varrho}+R_{\varrho \sigma \nu \lambda} Y_{\mu}^{\sigma}+R_{\mu \sigma \varrho \lambda} Y^{\sigma}{ }_{\nu}+R_{v \sigma \varrho \lambda} Y_{\mu}^{\sigma} .
$$

But the left-hand side of (5.14) is totally antisymmetric in $\mu, v$, and $\lambda$, so we may replace the right-hand side by its totally antisymmetric part in these indices. Rearrangement of these terms and use of a Bianchi identity yields Eq. (5.11). The left-hand side of (5.12) is identically

$$
\nabla_{[\mu} \nabla_{v]} Y_{\varrho \sigma}+\nabla_{[\varrho} \nabla_{\sigma]} Y_{\mu \nu}=0,
$$

reexpressing this using (5.11), rearranging terms and using the Bianchi identities yields (5.12). We have therefore

$$
K_{\mu ; \nu}=\frac{1}{2} R^{\varrho}{ }_{\nu} * Y_{\mu \varrho}+\frac{1}{4} R_{\mu \nu}{ }^{\varrho \sigma} * Y_{\varrho \sigma} .
$$

Contraction on $\mu$ and $\sigma$ of (5.12) gives (using the first Bianchi identity)

$$
Y_{(\varrho}^{\tau} R_{v) \tau}=0 \text {. }
$$

Thus using (5.8) in the case that $Y_{\mu \nu}$ has maximal rank or trivially if $g_{\mu \nu}$ is Ricci flat,

$$
* Y_{(\varrho}^{\tau} R_{v) \tau}=0 \text {. }
$$

Whence

$$
K_{(\mu ; v)}=0 .
$$

We are now in position to demonstrate the following interesting

Proposition 5.4. Any vacuum metric admitting a Yano tensor must be left and right type $D[25]$.

Proof. The left-hand side of (5.12) has the same symmetries as the Weyl tensor. We now adopt a manifestly $S O(3)_{L} \times S O(3)_{R}$ notation according to which the Weyl tensor is represented by 2 real symmetric trace free $3 \times 3$ matrices $C^{+}{ }_{i j}$ and the selfdual and antiself-dual parts of $Y_{\alpha \beta}$ by 2 real skew $3 \times 3$ matrices $Y^{ \pm}{ }_{i j}$. Then (5.12) reads

$$
C_{(i \mid k}^{ \pm} Y_{k \mid j)}^{ \pm}=0 \text {. }
$$

Taking in each equation in turn and using a basis in which $Y_{i j}$ has its canonical skew form, one finds that in this basis $C^{ \pm}{ }_{i j}$ are diagonal with 2 coincident eigenvalues. This is what is meant by type $D$.

Suppose that the metric is half-flat, then the Killing vector $K^{\alpha}$ is distinguished by the following 
Proposition 5.5. The primary Killing vector in a self-dual space admitting a Yano tensor is triholomorphic.

Proof. This follows immediately from (5.15).

From Propositions (5.3), (5.4), and (5.5) it follows that a non-trivial $Y_{\alpha \beta}$ cannot exist in the Atiyah-Hitchin metric [6] or double Taub-NUT. Since if a non-trivial $Y_{\alpha \beta}$ exists it is unique (up to a constant) and can be read off from the Weyl tensor, one knows that there are no non-trivial Yano tensors in Eguchi-Hanson. It seems clear that the other, $N>2$, multi-centre metrics are not type $D$, and indeed it seems to be true that the only non-singular Ricci flat type $D$ metrics are Schwarzschild, Kerr, Taub-Bolt [27] and the metric given in [28].

Yano tensors possess a number of other interesting properties which we shall now enumerate referring the reader to $[25,26]$ for proofs. One may check them in the specific case of Taub-NUT explicitly.

1) The Killing vector $K^{\alpha}$ is a symmetry of $Y_{\alpha \beta}$ :

$$
\underset{K^{\sigma}}{£} Y_{\alpha \beta}=0 \text {. }
$$

2) There exists an associated secondary Killing vector

$$
H^{\mu}=Y_{\lambda}^{\mu} Y_{\varrho}^{\lambda} K^{\varrho} .
$$

3) $H^{\mu}$ and $K^{\mu}$ commute and $H^{\mu}$ leaves $Y_{\mu \nu}$ invariant. In Taub-NUT $K^{\mu}, Y_{\mu}^{\lambda}$, and $H^{\mu}$ are $S O(3)$ invariant. In particular, this means that $H^{\mu}$ is triholomorphic, and hence (since it commutes with $K^{\mu}$ ) it must be proportional to $K^{\mu}$.

\section{The Simple Harmonic Oscillator}

To understand the significance of the hidden symmetries generated by the RungeLenz vector it is helpful to consider a simpler and rather well understood case: the isotropic oscillator in $N$ dimensions. In fact, our approach to the hidden symmetries will be to reduce the problem at constant energy and electric charge to one involving 4 isotropic oscillators. Much of this is elementary and well known but we wish to establish some notation and ideas that we shall use in Sect. 7. We shall begin classically and then proceed to the quantum case.

For any oscillator (isotropic or not) the symplectic group $\operatorname{Sp}(2 N, \mathbb{R})$ [sometimes called $S p(N, \mathbb{R})]$ of linear transformations of phase space leaving invariant the symplectic structure defined by:

$$
d p_{i} \wedge d q^{i}=\frac{1}{2} \Omega_{a b} d u^{a} \wedge d u^{b},
$$

with $i=1, \ldots, N, u^{a}=\left(q^{i}, p_{j}\right), a=1, \ldots, 2 N$, plays an important role, as does the orthogonal group of linear transformations leaving invariant the $2 \mathrm{~N}$-metric $g_{a b}$ defined by the Hamilton:

$$
H=\frac{1}{2} g_{a b} u^{a} u^{b}=\frac{1}{2} p_{i} p_{i}+\sum_{i=1}^{i=N} \frac{1}{2} \omega_{i}^{2} q^{i} q^{i}
$$

A third important group consists of those linear transformations leaving invariant the tensor $J_{b}^{a}$ defined by

$$
J^{a}{ }_{b}=\Omega^{a c} g_{c b},
$$


in terms of which the equations of motion are

$$
\frac{d u^{a}}{d t}=J_{b}^{a} u^{b}
$$

From (6.4) it is clear that this latter group takes classical solutions to classical solutions. In the special isotropic case in which all the frequencies $\omega_{i}$ are equal, one can rescale the time variable to make

$$
J^{a}{ }_{b} J_{c}^{b}=-\delta^{a}{ }_{c} .
$$

The group commuting with $J^{a}{ }_{b}$ may now be identified with $G L(N, \mathbb{C}), J^{a}{ }_{b}$ being a complex structure. The intersection of $G L(N, \mathbb{C})$ with $S O(2 N, \mathbb{R})$ is $S U(N)$ which leaves invariant the Hamilton and takes classical solutions to classical solutions. Thus the Hamiltonian symmetries of the system are much larger than the obvious $O(N)$ geometrical symmetry. The group $S U(N)$ is also the largest group of symplectic transformations leaving the Hamiltonian invariant. We may also express the special nature of the isotropic oscillator by saying that the endomorphism $J_{b}^{a}$ defined by (6.3) is an isometry of the metric $g_{a b}$. One may thus, in the isotropic case, introduce a complex notation manifesting these facts:

$$
b_{i}=\frac{1}{\sqrt{2}}\left(q^{i}+i p_{i}\right) .
$$

The equations of motion, Hamiltonian and symplectic forms thus become:

$$
\begin{gathered}
\frac{d b_{i}}{d t}=-i b_{i}, \\
H=b_{i} \bar{b}_{i}, \\
\Omega=i d \bar{b}_{i} \wedge d b_{i} .
\end{gathered}
$$

If $N$ is even and we may endow the configuration space with $N / 2$ complex coordinates $z^{\alpha}, \alpha=1, \ldots, N / 2$, such that

$$
z^{\alpha}=\frac{1}{\sqrt{2}}\left(q^{\alpha}+i q^{\alpha+\frac{N}{2}}\right),
$$

and

$$
p^{\alpha}=\frac{1}{\sqrt{2}}\left(p_{\alpha}-i p_{\alpha+\frac{N}{2}}\right),
$$

so that $p$ is canonically conjugate to $z$ and $\bar{p}$ to $\bar{z}$. Then one may introduce complex amplitudes

$$
\begin{aligned}
& b^{\alpha}+=\frac{1}{\sqrt{2}}\left(p^{\alpha}-i \bar{z}^{\alpha}\right), \\
& b_{-}{ }^{\alpha}=\frac{1}{\sqrt{2}}\left(\bar{p}^{\alpha}-i z^{\alpha}\right),
\end{aligned}
$$


and hence

$$
\dot{b}_{+}=-i b_{+} ; \quad \dot{b}_{-}=-i b_{-},
$$

and we have suppressed the $\alpha$ index in (6.14) as we shall do from now on. The symplectic structure is now:

$$
d p \wedge d z+d \bar{p} \wedge d \bar{z}=i\left(d \bar{b}_{+} \wedge d b_{+}+d \bar{b}_{-} \wedge d b_{-}\right) .
$$

Returning to the arbitrary $N$ case one has quantum mechanical operators $\hat{q}^{i}$ and $\hat{p}_{i}$ such that

$$
\begin{gathered}
{\left[\hat{q}^{i}, \hat{p}_{j}\right]=i \delta_{j}^{\prime},} \\
\hat{b}_{i}=\frac{1}{\sqrt{2}}\left(\hat{q}^{i}+i \hat{p}_{i}\right), \quad \hat{b}^{+}{ }_{i}=\frac{1}{\sqrt{2}}\left(\hat{q}^{i}-i \hat{p}_{i}\right) .
\end{gathered}
$$

( + denotes Hermitian conjugation with respect to the metric on the quantum mechanical Hilbert space.) The Hamiltonian is

$$
\hat{H}=\hat{b}^{+} \hat{b}_{i}+\frac{N}{2} .
$$

The 3 groups $S p(2 N, \mathbb{R}), S O(2 N, \mathbb{R})$, and $S U(N)$ in the classical problem also occur in the quantum mechanical problem. The set of invertible operators $\hat{O}$ obtained by exponentiating bilinears in the creation and annihilation operators $\hat{b}_{i}$ and $\hat{b}^{+}{ }_{i}$ act both on the quantum mechanical Hilbert space:

$$
|\psi\rangle \rightarrow \hat{O}|\psi\rangle
$$

and by conjugation on the algebra of operators $\hat{b}_{i}$ and $\hat{b}^{+}{ }_{i}$ :

$$
\hat{b}_{i} \rightarrow \widehat{O} b_{i} \hat{O}^{-1} \text {. }
$$

The largest such set commuting with the Hamiltonian $\hat{H}$ generates $G L(N, \mathbb{C})$. This is the analogue of the classical statement that the linear action of $G L(N, \mathbb{C})$ preserves the equation of motion (6.4). That is:

$$
\hat{O}=\exp \left(c_{i j} \hat{b}_{i} \hat{b}^{+}{ }_{j}\right)
$$

for arbitrary $c_{i j}$,

$$
\hat{b}_{i} \rightarrow\left(\exp c_{i j}\right) b_{j}
$$

The largest set of unitary operators obtained by exponentiating bilinears generates the group $\operatorname{Sp}(2 N, \mathbb{R})$. That is:

$$
\hat{O}=\exp \left(i c_{i j} \hat{b}_{i} b^{+}{ }_{j}+i\left(T_{i j} \hat{b}_{i} \hat{b}_{j}+\text { h.c. }\right)\right)
$$

with $c_{i j}=c_{j i}{ }^{*}$.

The intersection of these two set of operators is $U(N)$. Just as in the classical case the group $S O(2 N, \mathbb{R})$ plays no particularly important role and we shall not consider it further. The generator of the diagonal $U(1)$ is (up to zero point energy) the Hamiltonian. The remaining $S U(N)$ acts irreducibly on the set of states of fixed energy which have multiplicity $\left(\begin{array}{c}N+n-1 \\ n\end{array}\right)$, where $H=n+N / 2$. This represen- 
tation is not an irreducible representation of the $S O(N)$ subgroup generated by operators with $c_{i j}$ pure imaginary. The $S O(N)$ is the geometric symmetry and the $S U(N)$ is sometimes called the "degeneracy group." The group $\operatorname{Sp}(2 N, \mathbb{R})$ does not preserve the Hamiltonian and is generated by adding raising and lowering operators, e.g.

$$
\hat{b}_{1} \hat{b}_{1}, \quad \hat{b}_{1}^{\dagger} \hat{b}_{1}^{\dagger}
$$

which generate the algebra of $S U(1,1)$, sometimes called "the spectrum generating algebra":

$$
\begin{gathered}
{\left[\hat{b}_{1}, \hat{b}_{1}, \hat{H}\right]=2 \hat{b}_{1} \hat{b}_{1},} \\
{\left[\hat{b}^{+}{ }_{1} \hat{b}^{+}{ }_{1}, \hat{H}\right]=} \\
{\left[\hat{b}_{1} \hat{b}_{1}, \hat{b}_{1}^{\dagger} \hat{b}_{1}^{\dagger}\right]=4 \hat{b_{1}^{\dagger}} \hat{b}_{1}^{\dagger},}
\end{gathered}
$$

Notice that the operator $\hat{b}_{1}^{\dagger} \hat{b}_{1}^{\dagger}$ raises the energy by 2 units. In fact, the set of states with even $n$ and those with odd $n$ fill out an irreducible representation of $\operatorname{Sp}(2 N, \mathbb{R})$. If $N$ is even we may, as in the classical case, introduce the complex coordinates $z$ and the \pm notation. In the Schrödinger representation:

$$
\begin{gathered}
p=-i \frac{\partial}{\partial z}, \\
\bar{p}=-i \frac{\partial}{\partial \bar{z}}, \\
\hat{b}_{+}=\frac{-i}{\sqrt{2}}\left(\frac{\partial}{\partial z}+\bar{z}\right) ; \quad \hat{b}_{-}=\frac{-i}{\sqrt{2}}\left(\frac{\partial}{\partial \bar{z}}+z\right) ; \\
\hat{b}_{+}^{+}=\frac{-i}{\sqrt{2}}\left(\frac{\partial}{\partial \bar{z}}-z\right) ; \quad \hat{b}_{-}^{+}=\frac{-i}{\sqrt{2}}\left(\frac{\partial}{\partial z}-\bar{z}\right) .
\end{gathered}
$$

The Hamiltonian is thus:

$$
\begin{aligned}
\hat{H} & =\left(\hat{b}_{+}{ }^{+} \hat{b}_{+}+\hat{b}_{-}+\hat{b}_{-}+\frac{N}{2}\right) \\
& =-\frac{\partial^{2}}{\partial z \partial \bar{z}}+z \bar{z}
\end{aligned}
$$

Note that the generators of $S U(N)$ and $\operatorname{Sp}(2 N, \mathbb{R})$ are in general second order differential operators acting on functions $\Phi(z, \bar{z})$ and unitary with respect to the norm:

$$
\int \bar{\Phi} \Phi d^{N / 2} z d^{N / 2} \bar{z}
$$

\section{The Emergence of $S U(2,2)$}

The harmonic oscillator described in Sect. 6 is relevant for our problem only at constant electric charge, corresponding to the Killing vector $q^{\alpha}$. For the time being 
we shall remain with the case $N$ an arbitrary even positive integer. The $U(1)$ action

$$
\begin{gathered}
z \rightarrow e^{i \theta} z, \\
p \rightarrow e^{-i \theta} p,
\end{gathered}
$$

preserves the symplectic form (6.15). The action (7.1), (7.2) is that induced from the action on the configuration space whose infinitesimal generator is

$$
\frac{\partial}{\partial \psi}=i\left(z \frac{\partial}{\partial z}-\bar{z} \frac{\partial}{\partial \bar{z}}\right) \text {. }
$$

The simplectomorphism (7.1), (7.2) is generated by

$$
Q=i(z p-\bar{z} \bar{p})=2 q \text {. }
$$

The action (7.1) may be written as

$$
u^{a} \rightarrow\left(\exp \theta Q_{b}^{a}\right) u^{b}
$$

where $Q^{a}{ }_{b}$ is normalized so that

$$
Q^{a}{ }_{b} Q^{b}{ }_{c}=-\delta^{a}{ }_{c} .
$$

Thus the $U(1)$ action defines a second complex structure on the phase space. In terms of the coordinates $b_{ \pm}, Q$ is given by

$$
\left(\bar{b}_{+} b_{+}-\bar{b}_{-} b_{-}\right)
$$

while $J_{b}^{a}$ corresponds to the Hamiltonian:

$$
\left(\bar{b}_{+} b_{+}+\bar{b}_{-} b_{-}\right) \text {. }
$$

Because the complex structures $J$ and $Q$ commute the space splits into a direct sum (labelled in our case by \pm ) restricted to which $Q= \pm J$, respectively. Thus we obtain no further complex structures from $J$ and $Q$.

The group commuting with $Q$ is isomorphic to $G L(N, \mathbb{C})$, we shall call it $G L_{Q}(N, \mathbb{C})$, but it is not, of course, the same $G L(N, \mathbb{C})$ as that commuting with $J$. The subgroup of $G L_{Q}(N, \mathbb{C})$ commuting with the symplectic structure is $U\left(\frac{N}{2}, \frac{N}{2}\right)$, i.e. the pair $\Omega_{a b}, Q^{a}{ }_{b}$ define a Hermitian structure with signatures $N / 2, N / 2$ just as the pair $\Omega_{a b}, J_{b}^{a}$ define a positive definite Hermitian structure. The intersection of the group preserving both the Hamiltonian and the electric charge is $S U(N / 2) \times S U(N / 2)$. In the \pm basis this may be written as

$$
\left(\begin{array}{cc}
U_{+} & 0 \\
0 & U_{-}
\end{array}\right) ; \quad U_{ \pm} \in S U\left(\frac{N}{2}\right) \text {. }
$$

The charge acts as

and the Hamiltonian as

$$
\begin{gathered}
\left(\begin{array}{cc}
e^{\frac{i \theta}{2}} & 0 \\
0 & e^{\frac{-i \theta}{2}}
\end{array}\right) \\
\left(\begin{array}{cc}
e^{i t} & 0 \\
0 & e^{i t}
\end{array}\right)
\end{gathered}
$$


The discussion above has been classical. However, the reader may readily generalize to the quantum mechanical case by replacing the quantities $b_{ \pm}$in (7.7) by their quantum analogues.

Since the Hamiltonian (7.8) played no role in defining the $U(N / 2, N / 2)$ action, it follows that we can use this construction for any phase space of the form $T^{*}(M)$, where $M$ is a complex manifold with a holomorphic $U(1)$ action which is linear. For example in the case of Eguchi-Hanson in $\zeta^{1}$ and $\zeta^{2}$ of (2.2.3) and (2.2.4) we have a natural symplectic action of $S U(2,2)$ on the cotangent bundle.

From now on we restrict our attention to $N=4$ :

The $\bar{z}$ and $p$ carry a complex 4-dimensional representation of $S U(2,2)$ which is in fact isomorphic with the "twistor" representation or equivalently the Weyl spinors of $S O(4,2)$. In what follows we shall use some work of Mack and Todorov [29]. We define a 4-component vector by:

If

$$
\phi=\left(\begin{array}{c}
\bar{z} \\
i p
\end{array}\right) .
$$

$$
\beta=\left(\begin{array}{cc}
0 & \mathbb{1}_{2} \\
\mathbb{1}_{2} & 0
\end{array}\right),
$$

the $S U(2,2)$ Hermitian inner product is

$$
\phi^{+} \beta \phi=-i(z p+\bar{p} \bar{z}),
$$

in terms of which the symplectic form (6.15) is

$$
i d \phi^{+} \beta \wedge d \phi \text {. }
$$

Note that the complex conjugate appears in $(7.12)$ because the $S U(2,2)$ is holomorphic with respect to the complex structure $Q^{a}{ }_{b}$ and not with respect to the original $J_{b}^{a}$. Following Mack and Todorov we may identify the generators of $S O(4,2)$ by using the following representation of the 4-dimensional $\gamma$-matrices:

$$
\gamma_{0}=\beta ; \quad \gamma_{i}=\left(\begin{array}{cc}
0 & -\sigma_{i} \\
\sigma_{i} & 0
\end{array}\right) ; \quad \gamma_{5}=\left(\begin{array}{cc}
\mathbb{1}_{2} & 0 \\
0 & -\mathbb{1}_{2}
\end{array}\right),
$$

whence the generators of $S O(4,2) \gamma_{A B}, A=0,1,2,3,4,5$ in the twistor representation are:

$$
\begin{gathered}
\gamma_{\mu \nu} \equiv \frac{1}{4}\left[\gamma_{\mu}, \gamma_{\nu}\right] \equiv M_{\mu \nu}, \\
\gamma_{5 b} \equiv \frac{1}{2} \gamma_{5}=\frac{1}{2} \gamma_{0} \gamma_{1} \gamma_{2} \gamma_{3} \equiv D, \\
\gamma_{\mu S} \equiv \frac{1}{2} i \gamma_{\mu} \gamma_{5} \equiv \frac{1}{2}\left(p_{\mu}-K_{\mu}\right), \\
\gamma_{\mu 6} \equiv \frac{1}{2} \gamma_{\mu} \equiv \frac{1}{2}\left(p_{\mu}+K_{\mu}\right) .
\end{gathered}
$$

The labelling above allows a convenient identification of the Poincare and de Sitter subgroups. So far the discussion has been classical. Quantum mechanically we may replace

$$
p \rightarrow-i \frac{\partial}{\partial z}
$$


in (7.12). The quantum operators $\hat{\phi}$ then satisfy the "twistor commutation relations" [30]

$$
\begin{gathered}
{\left[\tilde{\hat{\phi}}^{\dagger}, \hat{\phi}\right]=1,} \\
{[\hat{\phi}, \hat{\phi}]=0,}
\end{gathered}
$$

and the electric charge $\hat{q}$ is given

$$
\hat{q}=\frac{1}{2}\left(\tilde{\hat{\phi}}^{\dagger} \hat{\phi}+2\right)
$$

and the generators of $S O(4,2)$ are

$$
J_{A B}=\tilde{\hat{\phi}}^{\dagger} \gamma_{A B} \hat{\phi}
$$

where $\sim \uparrow$ denotes Hermitian conjugation transposition and post multiplication by $\beta$ (Dirac conjugation). Note that $\hat{q}$ plays the role of "helicity" in the conformal language.

Having set up this formalism one may write the generators as (in general) second order differential operators acting on functions of $z$ and $\bar{z}$, which are selfadjoint with respect to the norm (6.32). The reader is referred to Mack-Todorov for the explicit expressions which they have also expressed in the coordinates $\psi, \underline{r}$ used earlier. The relation between their coordinates $\zeta^{i}$ and $\alpha$ is

$$
\begin{aligned}
\alpha & =\psi, \\
\xi^{\prime} & =-r \sin \theta \cos \phi, \\
\xi^{2} & =-r \sin \theta \sin \phi, \\
\xi^{3} & =-r \cos \theta .
\end{aligned}
$$

The reader should take the upper signs in [29]. The first order operators $M_{i j}$ are SO(3) Killing vectors for Taub-NUT.

So far we have not introduced the Hamiltonian. Classically, this is given by

$$
H=\frac{4 p \bar{p}}{|z|^{2}-4}-\frac{1|z|^{2}-8}{4|z|^{2}-4}|z p-\bar{z} p|^{2},
$$

and quantum mechanically by

$$
\hat{H}=-\frac{1}{\left(|z|^{2}-4\right)}\left\{-4 \frac{\partial^{2}}{\partial z \partial \bar{z}}+\frac{1}{4}\left(\left(|z|^{2}-8\right) \frac{\partial^{2}}{\partial \psi^{2}}\right)\right\} .
$$

Note that $\hat{H}$ is self-adjoint with respect to the Hilbert space pseudo metric:

$$
\int d^{2} z d^{2} \bar{z}\left(|z|^{2}-4\right) \bar{\Phi} \Phi
$$

which coincides with the Riemannian measure on Taub-NUT (with a negative mass). If we considered the positive mass Taub-NUT metric the factor $|z|^{2}-4$ would be replaced by $|z|^{2}+4$ which gives a positive definite inner product on the Hilbert space.

The subgroup of $S U(2,2)$ commuting with the Hamiltonian is the diagonal $S U(2)$ subgroup of isometries. At any given fixed energy and electric charge, classically satisfying $\varepsilon<s^{2} / 4$, i.e. corresponding to the bound states, we can find (as explained in [6]) a further $S U(2)$ subgroup which leaves invariant the submanifold 
of phase space with these constant values. Quantum mechanically we can find a further $S U(2)$ subgroup of $S U(2,2)$ which leaves the subspace of constant energy and constant electric charge fixed. Thus classically:

$$
\begin{aligned}
& z \rightarrow \frac{1}{2}\left(\bar{u}_{+}+u_{-}\right) z-\frac{\left(u_{+}-\bar{u}_{-}\right)}{\left(s^{2}-4 \varepsilon\right)^{1 / 4}} i p, \\
& p \rightarrow \frac{1}{2}\left(\bar{u}_{+}+u_{-}\right) p-i \frac{\left(\bar{u}_{+}-u_{-}\right)}{4}\left(s^{2}-4 \varepsilon\right)^{1 / 2} \bar{z},
\end{aligned}
$$

where $U_{+}$and $U_{-}$are $S U(2)$ matrices.

Thus for definite energy and definite electric charge we have an $S U(2) \times S U(2)$ symmetry taking classical orbits to classical orbits. This admits an elegant geometrical description on phase space:

Define

$$
\begin{aligned}
& a_{+}=\frac{i \bar{p}}{\left(s^{2}-4 \varepsilon\right)^{1 / 4}}+\frac{\left(s^{2}-4 \varepsilon\right)^{1 / 4}}{2} z, \\
& a_{-}=\frac{i p}{\left(s^{2}-4 \varepsilon\right)^{1 / 4}}+\frac{\left(s^{2}-4 \varepsilon\right)^{1 / 4}}{2} \bar{z},
\end{aligned}
$$

then the charge and Hamiltonian constraints become

$$
\begin{gathered}
s=\frac{1}{2}\left(\bar{a}_{-} a_{-}-\bar{a}_{+} a_{+}\right), \\
\frac{2 s^{2}-4 \varepsilon}{\left(s^{2}-4 \varepsilon\right)^{1 / 2}}=\left(\bar{a}_{-} a_{-}+\bar{a}_{+} a_{+}\right) .
\end{gathered}
$$

The symplectic form is

$$
i\left(d \bar{a}_{+} \wedge d a_{+}+d \bar{a}_{-} \wedge d a_{-}\right) .
$$

The functions $q$ and $H$ generate the actions:

$$
\begin{aligned}
a_{+} \rightarrow e^{-i \frac{\theta}{2}} a_{+} ; & a_{-} \rightarrow e^{-i \frac{\theta}{2}} a_{-}, \\
a_{+} \rightarrow e^{i t} a_{+} ; & a_{-} \rightarrow e^{i t} a_{-},
\end{aligned}
$$

respectively. Equations (7.30) and (7.31) are equivalent to

$$
\frac{1}{4}\left|a_{ \pm}\right|^{2}=\left|\underline{A}_{ \pm}\right|^{2}
$$

where $\left|\underline{A}_{ \pm}\right|^{2}$ are the functions of $\varepsilon$ and $s$ given by (3.13).

Equations (7.35) define two 3-spheres, factoring by the action of (7.33), and (7.34) is equivalent to Hopf fibering these 3-spheres to give two 2-spheres. In terms of $a_{ \pm}$the action (7.27) reads

$$
\left(a_{+}, a_{-}\right) \rightarrow\left(u_{+} a_{+}, u_{-} a_{-}\right) .
$$

Quantum mechanically we must proceed in a similar way. We may (restricted to the subspace of states for fixed $\hat{q}=s$ and $\hat{H}=\varepsilon$ ) define [using formulas (7.28) and (7.29)] the bilinears in the $\hat{a}$ 's and $\hat{a}^{+}$'s which restricted to the subspace of constant 
energy and electric charge generate the action of $S U(2) \times S U(2)$. This action is irreducible on this subspace, carrying the $\left(\frac{n+s-1}{2}, \frac{n-s-1}{2}\right)$ representation of $S U(2) \times S U(2)$ with degeneracy $n^{2}-s^{2}$, where $n>|s|$ is the quantum number introduced in [5].

\section{Large Distance Fluctuations Around B.P.S. Multi-Monopoles}

In this section we shall discuss the fluctuations of the gauge and Higgs fields about an arbitrary solution of the Bogomolnyi equations. It was shown in [31] that these are all governed by a single linear equation for a pseudo scalar Higgs field $p$ which must satisfy:

$$
-D_{i} D^{i} p+[\Phi,[\Phi, p]]=\omega^{2} p
$$

where $D_{i}$ denotes covariant differentiation with respect to the background connection and $\Phi$ is the background Higgs field.

At large distances it seems reasonable to believe that the background configuration will tend (in a suitable gauge) to one in which

$$
\begin{aligned}
& \underline{A}^{a} \sim \delta_{3}^{a} \underline{A}, \\
& \Phi^{a} \sim \delta_{3}^{a} \phi,
\end{aligned}
$$

where $\underline{A}$ and $\phi$ satisfy the Abelian Bogomolnyi equations,

$$
\underline{\operatorname{curl}} \underline{A}=\underline{\operatorname{grad}} \phi,
$$

which coincide, of course, with Eq. (2.1.3). For example in the single monopole case the solution of the Bogomolnyi equations is

$$
\begin{gathered}
A^{a}=\varepsilon^{a}{ }_{i j} \frac{d x^{i} x^{j}}{r}\left(\frac{1}{r}-\frac{1}{\sinh r}\right), \\
\Phi^{a}=\frac{x^{a}}{r}\left(\frac{1}{r}-\operatorname{coth} r\right) .
\end{gathered}
$$

At large distances we may drop the exponential terms to get the Wu-Yang limiting form

$$
\begin{gathered}
A^{a} \sim \varepsilon^{a}{ }_{i j} \frac{d x^{i} x^{j}}{r^{2}}, \\
\Phi^{a} \sim \frac{x^{a}}{r}\left(\frac{1}{r}-1\right) .
\end{gathered}
$$

Under the gauge transformation by

$$
g_{N}=\left(\begin{array}{cc}
\cos \frac{\theta}{2}, & -\sin \frac{\theta}{2} e^{-i \phi} \\
\sin \frac{\theta}{2} e^{+i \phi}, & \cos \frac{\theta}{2}
\end{array}\right)
$$


or

$$
g_{S}=g_{N}(\pi-\theta, \phi+\pi),
$$

one finds that the solutions of (8.4) are

$$
\begin{gathered}
A_{N}=-2 \sin ^{2} \frac{\theta}{2} d \phi, \\
\phi_{N}=-\left(1-\frac{1}{r}\right),
\end{gathered}
$$

or

$$
\begin{gathered}
A_{S}=-2 \cos ^{2} \frac{\theta}{2} d \phi, \\
\phi_{S}=+\left(1-\frac{1}{r}\right) .
\end{gathered}
$$

The pair $\left(A_{N}, \phi_{N}\right)$ respectively $\left(A_{S}, \phi_{S}\right)$ are regular in the northern, respectively southern hemisphere.

The fact that both $A^{a}$ and $\Phi^{a}$ have only a 3-component - which would not be true if we used the same gauge transformation on the full interior B.P.S. configuration - reflects a greater symmetry in the asymptotic $\mathrm{Wu}$-Yang limit than in the full B.P.S. case. The B.P.S. monopole is $S O(3)$ invariant. The Wu-Yang limit has an additional $U(1)$ (rotation about the 3 directions). This extra symmetry corresponds physically to electric charge. This charge is not conserved for deep scattering against the B.P.S. monopole but it is conserved in the Wu-Yang limit. This situation closely parallels the case of monopole-monopole interactions which are only charge conserving in the asymptotic large distance limit.

Let us now consider the fluctuations around a general Abelian solution. We may expand the pseudoscalar field $P$ in a basis for the Lie algebra:

$$
p=\frac{1}{2 i}\left(p^{3} \tau_{3}+p^{+} \tau_{+}+p^{-} \tau_{-}\right)
$$

and find that Eqs. (8.1) become

$$
-(\underline{\nabla}-i s \underline{A})(\underline{\nabla}-i s \underline{A}) p^{a}+s^{2} \phi^{2} p^{a}=\omega^{2} p^{a},
$$

where $s=0,+1$ or -1 for $a=3,+1$, or -1 , respectively.

If the reader compares Eq. (7.16) with (2.20) he will accede to the following

Proposition 8.1. The fluctuations around the Abelian Bogomolnyi equations are governed by the equation

$$
-\nabla_{\alpha} \nabla^{\alpha} p^{a}=\frac{\omega^{2}}{V} p^{a}
$$

where $\nabla_{\alpha}$ is the covariant derivative on the self-dual metric generated by $\phi$ with $\tau$-dependence $\exp +i s \tau, s=0, \pm 1$. 
Proposition (8.1) amplifies some rather cryptic remarks of Schonfeld [32]. See also [37]. For a harmonic function $\phi$ with one or two mass points Eq. (8.17) is a special case of Eq. (4.9) which satisfies condition (4.13). Thus we may by Proposition (4.1) solve Eqs. (8.16) by separation of variables. In the 2-monopole case this appears to be a new result. In the single monopole case the problem may be solved by the same method as used for the ordinary Laplacian or Taub-NUT and possesses additional conserved quantities. We have indicated in [6] how the Taub-NUT results may be transcribed to solve Eq. (8.17) in this case. Since there already exists an extensive discussion of the resulting solution in the literature [33] we shall not pursue this matter further here.

One amusing point in the 2-monopole case which does not seem to have been pointed out before is that since each centre gives rise to an attractive potential, Eq. (8.17) resembles the Schrödinger equation for the ionized Hydrogen molecule (with a magnetic term if $s=0$ ). Thus something akin to molecular energy levels and the possibility of molecular binding forces seems to arise.

\section{B.P.S. Monopole Scattering with a Wess-Zumino Term}

The quantum scattering of B.P.S. monopoles was described in [5] using the Schrödinger equation on the Atiyah-Hitchin metric. In fact, given a configuration space $\left(M, g_{\alpha \beta}\right)$ we may consider quantizing using as "wave functions" the sections of any line bundle over it [34]. Since $H_{2}$ (Atiyah-Hitchin, $\left.\mathbb{Z}\right)=\mathbb{Z}$ there is essentially a single integer's worth of such line bundles with a connection whose curvature is proportional to the generator of $H^{2}$. Explicitly if the self-dual Atiyah-Hitchin metric is written as

$$
d s^{2}=f^{2} d r^{2}+a^{2} \sigma_{1}^{2}+b^{2} \sigma_{2}^{2}+c^{2} \sigma_{3}^{2},
$$

then the closed 2-form

$$
F=d g \wedge \sigma_{1}+g \sigma_{2} \wedge \sigma_{3}
$$

is $\varepsilon$-self-dual iff

$$
\frac{d g}{d r}=-\varepsilon g f a /|b c|
$$

If $\varepsilon=+1$ this is normalizable. If $\varepsilon=-1$ this is not normalizable and is exact. Choosing $\varepsilon=+1$ and imposing the "Dirac quantization condition" we find that the curvature of the $p$ th line bundle is given by

$$
g=\frac{1}{2} p \exp -\int_{\pi}^{r} d r \frac{f a}{b|c|},
$$

$p= \pm 1, \pm 2, \ldots$. Away from $\tilde{\theta}=0$, or $\pi$ we have

$$
F=d A
$$

with

$$
A=g \sigma_{1}
$$


We may consider therefore the Schrödinger equation:

$$
-\left(\nabla_{\mu}-i A_{\mu}\right)\left(\nabla^{\mu}-i A^{\mu}\right) \Phi=\varepsilon \Phi .
$$

Physically, one might imagine that the $A_{\mu}$ might arise from "integrating over fermion fields" if for example one considered the B.P.S. monopole as a soliton of the $N=4$ super Yang-Mills theory. We shall not give a detailed justification here because in this paper we are merely interested in how inclusion of the vector potential $A_{\mu}$ changes the boundary conditions for the wave function $\Phi$ and hence its solution. The 2 -form $F$ decays exponentially fast away from the bolt. Thus $A_{\mu}$ would not appear in Eq. (9.7) in the asymptotic Taub-NUT region. However, it does affect the boundary conditions in Taub-NUT, since it determines the choice of eigenvalues of $s=-i \frac{\partial}{\partial \psi}$. Near the bolt the vector potential (5.6) becomes

$$
A \sim \frac{p}{2}(d \tilde{\psi}+\cos \tilde{\theta} d \tilde{\phi})
$$

and the metric (9.1)

$$
d s^{2} \sim d s^{2}+4(r-\pi)^{2}(d \tilde{\psi}+\cos \tilde{\theta} d \tilde{\phi})^{2}+\pi^{2}\left(d \tilde{\theta}^{2}+\sin ^{2} \widetilde{\theta} d \tilde{\phi}^{2}\right)
$$

with $0 \leqq \tilde{\psi} \leqq \pi$. The vector potential (9.8) is singular at the bolt $r=\pi$. We must move to a well-behaved gauge $A^{i}$ such that the gauge transformed section $\Phi^{\prime}$ is single valued.

A suitable gauge is given by:

$$
A^{\prime}=\sin \tilde{\theta} d \tilde{\phi}=A-\frac{p}{2} d(\tilde{\psi}+\cos \tilde{\theta} \tilde{\phi})
$$

Thus

$$
\Phi^{\prime}=\Phi \exp -\frac{i p}{2}(\tilde{\psi}+\cos \tilde{\theta} \tilde{\phi})
$$

If $\Phi^{\prime}$ is single valued under $\tilde{\psi} \rightarrow \tilde{\psi}+\pi$, i.e.

$$
I_{1} \Phi^{\prime}=\Phi^{\prime}
$$

with $I_{1}$ defined by (3.24) of [5] we have

$$
I_{1} \Phi=(-1)^{p} \Phi .
$$

Equation (9.14) differs from that used in [5] if $p \in 2 \mathbb{Z}+1$. Expressed in terms of the asymptotic Euler angles of the Taub-NUT metric $I_{1}$ is the map ((3.17) of [5])

$$
I_{1}: \quad \theta \rightarrow \pi-\theta ; \quad \phi \rightarrow \pi+\phi ; \quad \psi \rightarrow-\psi ; \quad x \rightarrow x .
$$

If $p \in 2 \mathbb{Z}+1$, then Eq. (9.14) has profound physical consequences. Consider to begin with the scattering. In parabolic coordinates the wave function satisfies

$$
\Phi(r+z, r-z, \phi, \psi)=(-1)^{p} \Phi(r-z, r+z, \phi+\pi,-\psi)
$$


(this replaces (7.19) of [5]). If $s=0$, i.e. 2 neutral monopoles, then (9.16) shows that they scatter like fermions rather than bosons.

As for the bound states, the appropriate angular functions replacing Eq. (6.25) of $[5]$ are

$$
W_{s m}^{j} \equiv e^{i m \phi}\left[e^{i s \psi} d_{s m}^{j}(\theta)+(-1)^{j+p} e^{-i s \psi} d^{j}{ }_{-s m}(\theta)\right],
$$

which still satisfy

$$
I_{3} W^{j}{ }_{s m}=(-1)^{s} W^{j}{ }_{s m}
$$

\section{Killing Tensors and $\sigma$-Models}

One reason for being interested in hyperKähler metrics is that they provide target spaces for finite non-linear models. It is therefore of interest to ask what does the existence of a Killing tensor on the target manifold imply for the $\sigma$-model. The short answer is unfortunately nothing. If $x^{\alpha}(u)$ are thought of as maps from some spacetime with coordinates $u^{i}, i=1, \ldots, n$ and metric $h_{i j}(u)$ it is well known that Killing fields $K^{\alpha}(x)$ on the target manifold give rise to conserved currents $J_{i}$ on the spacetime via the formula

$$
J_{i}=g_{\alpha \beta} K^{\alpha} \frac{\partial x^{\beta}}{\partial u^{i}}
$$

The conserved stress tensor $T_{i j}$ is given by:

$$
T_{i j}=g_{\alpha \beta} \frac{\partial x^{\alpha}}{\partial u^{i}} \frac{\partial x^{\beta}}{\partial u^{j}}-\frac{1}{2} h_{i j} g_{\alpha \beta} \frac{\partial x^{\alpha}}{\partial u^{r}} \frac{\partial x^{\beta}}{\partial u^{s}} h^{r s} .
$$

It is natural therefore to replace $g_{\alpha \beta}$ in $(10.2)$ by an arbitrary symmetric tensor $K_{\alpha \beta}$ :

$$
s_{i j}=K_{\alpha \beta} \frac{\partial x^{\alpha}}{\partial u^{i}} \frac{\partial x^{\beta}}{\partial u^{j}}-\frac{1}{2} h_{i j} K_{\alpha \beta} \frac{\partial x^{\alpha}}{\partial u^{r}} \frac{\partial x^{\beta}}{\partial u^{s}} h^{r s} .
$$

One finds that $s_{i j}$ is conserved iff

$$
K_{\alpha \beta ; \gamma}=0 .
$$

Of course, Eq. (10.4) implies the Killing tensor equation (3.17) but it is much stronger. In particular, it implies that $g_{\alpha \beta}$ is reducible (cf. [35]), i.e. a metric product of 2 lower dimensional manifolds. Thus the Killing tensors on Taub-NUT [which do not satisfy (4)] do not give rise to interesting conserved quantities for a $\sigma$-model. This result is somewhat reminiscent of a general result of Coleman and Mandula [36] to the effect that conserved charges which are not Lorentz invariant and which arise from local currents (local in spacetime) are incompatible with the general principles of field theory.

Acknowledgements. We should like to thank B. Carter, N. Hitchin, P. Horvathy, and N. Woodhouse for useful discussions. 


\section{References}

1. Jackiw, R.: Rev. Mod. Phys. 49, 681 (1977)

2. Manton, N.S.: Phys. Lett. 110 B, 54 (1982)

3. Atiyah, M.F., Hitchin, N.J.: Phys. Lett. 107 A, 21 (1985); Phil. Trans. Roy. Soc. Lond. A 315, 459 (1985); The geometry and dynamics of magnetic monopoles. Princeton, NJ: Princeton University Press (in press)

4. Manton, N.S.: Phys. Lett. 154 B, 397 (1985); (E) 157 B, 475 (1985)

5. Gibbons, G.W., Manton, N.S.: Nucl. Phys. B 274, 183-224 (1986)

6. Gibbons, G.W., Ruback, P.: Phys. Lett. B 188, 226 (1986)

7. Atiyah, M.F., Hitchin, N.J., Singer, I.M.: Proc. Roy. Soc. Lond. A 362, 425 (1978)

8. Page, D.N.: Unpublished notes Yuille, A.: Ph.D. Thesis, Cambridge University

9. Page, D.N.: Phys. Lett. 85 B, 369-372 (1979)

10. Gibbons, G.W., Freedman, D.Z.: In: Superspace and supergravity. Hawking, S.W., Rocek, M. (eds.). Cambridge: Cambridge University Press 1981

11. Besse, A.L.: Einstein manifolds. Berlin, Heidelberg, New York: Springer 1987

12. Gibbons, G.W., Pope, C.N.: Commun. Math. Phys. 66, 267 (1979)

13. Valent, G., Olivier, D.: PAR-LPTHE 86/22, unpublished

14. Fehér, L.G., Horvathy, P.A.: Phys. Lett. 183 B, 182-186 (1987)

15. Carter, B.: Phys. Rev. D 16, 3395-3414 (1977)

16. Woodhouse, N.M.J.: Commun. Math. Phys. 44, 9 (1975)

17. Benenti, S., Francaviglia, M.: In: General relativity and gravitation, Vol. I. Held, A. (ed.). New York: Plenum 1980

18. Oyvind Burrau: Det. Kgl. Danske Vid. Selskab. 7, 1 (1927)

19. Schwinger, J.: J. Math. Phys. 5, 1606-1608 (1964)

20. Hostler, L.: J. Math. Phys. 5, 591-595 (1964)

21. Hostler, L., Pratt, R.H.: Phys. Rev. Lett. 10, 469-470 (1963)

22. McIntosh, H.V.: Symmetry and degeneracy. In: Group theory and its applications. II. Locbl (ed.). New York: Academic Press

23. Alliluev, S.P., Matveenko, A.V.: Sov. Phys. J.E.T.P. 24, 1260-1264 (1967)

24. Bargmann, V.: Z. Phys. 99, 576 (1936)

25. Dietz, W., Rudinger, R.: Proc. Roy. Soc. A 375, $361-378$ (1981); A 381, 315-322 (1982)

26. Carter, B.: J. Math. Phys. 28, 1535-1540 (1987)

27. Page, D.N.: Phys. Lett. 78 B, 249 (1978)

28. Gibbons, G.W., Perry, M.: Phys. Rev. D 22, 313-321 (1980)

29. Mack, G., Todorov, I.: J. Math. Phys. 10, 2078 (1969)

30. Penrose, R.: In: Quantum gravity - an Oxford symposium. Isham, C.J., Penrose, R., Sciama, D. (eds.). Cambridge: Cambridge University Press 1974

31. D’Adda, A., Horsley, R., Di Vecchia, P.: Phys. Lett. 76 B, $298-302$ (1978)

32. Schonfeld, J.: J. Math. Phys. 21, 2528 (1980)

33. Biebl, K.J., Wolf, J.: Nucl. Phys. B 279, 571 (1987)

34. Simms, D.J., Woodhouse, N.M.J.: Lectures on geometric quantization. Lecture Notes in Physics, Vol. 53. Berlin, Heidelberg, New York: Springer 1976

35. Hoenselaers, C.: Article in: Unified field theories of more than 4 dimensions, including exact solutions. De Sabatta, V., Schmutzer, E. (eds.). Singapore: World Scientific 1983

36. Coleman, S., Mandula, J.: Phys. Rev. 159, 1251 (1967)

37. Feher, L.G.: J. Phys. A 19, 1259-1270 (1980)

Feher, L.G.: In: Non-perturbative methods in Q.F.T. Horvath, Z., Palla, L., Palkos, A. (eds.). Singapore: World Scientific 1987

38. Gibbons, G.W., Olivier, D., Ruback, P.J., Valent, G.: Nucl. Phys. B (in press)

Communicated by L. Alvarez-Gaumé 
